CERN-PPE/96-134

2 October 1996

\title{
ENHANCED AND QUENCHED B(E1) TRANSITION RATES BETWEEN PARITY DOUBLET BANDS IN ${ }^{227}$ Ra
}

\author{
A.J. Aas ${ }^{1)}$, M.J.G. Borge ${ }^{3)}$, B. Fogelberg ${ }^{2)}$, I.S. Grant ${ }^{4)}$, K. Gulda ${ }^{5)}$, E. Hagebo ${ }^{1)}$,

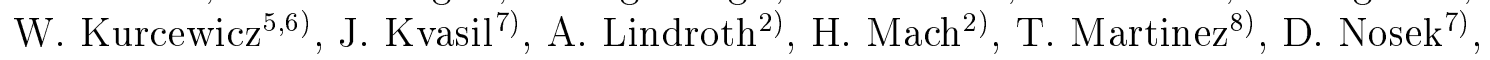 \\ B. Rubio ${ }^{8)}$, J.F. Smith ${ }^{9)}$, K. Steffensen ${ }^{1)}$, J.L. Tain ${ }^{8)}$, O. Tengblad ${ }^{3,6)}$, \\ T.F. Thorsteinsen ${ }^{10)}$, \\ and the ISOLDE Collaboration.
}

\begin{abstract}
The fast timing $\beta \gamma \gamma(\mathrm{t})$ method has been used to measure level lifetimes in the parity doublet bands in ${ }^{227} \mathrm{Ra}$ populated in the $\beta^{-}$decay of ${ }^{227} \mathrm{Fr}$. In particular, $T_{1 / 2}=254(9) \mathrm{ps}, 236(30) \mathrm{ps}, \leq 41 \mathrm{ps}$, and 16(13) ps have been measured for the $3 / 2^{-}$and $5 / 2^{-}$members of the $K^{\pi}=3 / 2^{-}$band and for the $1 / 2^{-}$and $3 / 2^{-}$ members of the $K^{\pi}=1 / 2^{-}$band, respectively. These results indicate surprisingly fast $B(E 1)$ rates in this octupole transitional nucleus with the average $\left|D_{0}\right|$ value of $\geq 0.11 \mathrm{e} \cdot \mathrm{fm}$ for the $K^{\pi}=1 / 2^{-}$band and $\left|D_{0}\right|=0.10 \mathrm{e} \cdot \mathrm{fm}$ for $K^{\pi}=3 / 2^{-}$band. The general enhancement (and occasional quenching) of $E 1$ intra-doublet and some enhanced extra-doublet transitions in ${ }^{227} \mathrm{Ra}$ are interpreted within the quasiparticleplus-phonon model with the inclusion of Coriolis coupling. Similar calculations, performed for ${ }^{223} \mathrm{Ra}$, are found to be in good agreement with the experimental data.
\end{abstract}

\section{(IS322)}

\section{(Accepted for publication in Nuclear Physics A)}

1) Department of Chemistry, University of Oslo, P.O. Box 1033, Blindern, N-0315 Oslo, Norway

2) Department of Neutron Research, University of Uppsala, S-61182 Nyköping, Sweden

3) Insto. De Estructura de la Materia, CSIC, E-28006 Madrid, Spain

4) The Schuster Laboratory, Manchester University, Manchester M13 9PL, UK

5) Department of Physics, University of Warsaw, Pl-00 681 Warsaw, Poland

6) PPE Division, CERN, CH-1211 Geneva 23, Switzerland

7) Department of Nuclear Physics, Charles University, V Holesovičkach 2, 18000 Prague 8, Czech Republic

8) Insto. de Física Corpuscular, CSIC-Univ. Valencia, E-46100 Burjassot, Spain

9) Oliver Lodge Laboratory, University of Liverpool, P.O.Box 147, Liverpool, L69 3BX, UK

10) Department of Physics, University of Bergen, N-5007 Bergen, Norway 
The structure of Ra and Th nuclei evolves from spherical at $N=126$ to quadrupole deformed near $N \sim 142$ as they span the central part of the octupole collective region at $N \sim 136$. This strategic location makes them an ideal testing ground for the strong interplay of quadrupole and octupole collectivities which uniquely characterize the heavy actinides. In contrast to the energy systematics of the low-lying positive and negative parity bands, which in Ra and Th show a close similarity [1], the systematics of their dipole moments $D_{0}$ (derived from $B(E 1)$ rates) is quite different. In heavy Th nuclei, there is a smooth decrease of the $D_{0}$ values from a maximum near $N \sim 134$ to a small value near $N \sim 140$, while the Ra systematics show strong fluctuations with the unique phenomenon of $D_{0}$ quenching at $N \sim 136[1,2]$.

The recent calculations $[1,2]$ correctly reproduce the general features of the energy and $D_{0}$ systematics. However, the microscopic calculations of Egido and Robledo [1] predict fast $B(E 1)$ rates for ${ }^{228,230} \mathrm{Ra}(N=140,142)$, while the schematic model calculations of Butler and Nazarewicz [2] seem to imply lowering of the dipole moment for heavy Ra isotopes and a possible strong configuration-dependence of $D_{0}$ for selected odd nuclei. These predictions remain to be verified.

The $B(E 1)$ systematics for the low-lying states, which are the crucial testing ground for the theoretical predictions, remain fairly incomplete in this region [2], particularly for the heavier transitional nuclei. Despite a few direct measurements (e.g. from Coulomb excitation or lifetime measurements), most $B(E 1)$ rates have been estimated from $B(E 1) / B(E 2)$ branching ratios by assuming that these nuclei are good rotors, having a constant quadrupole moment through the rotational band, equal to that of the groundstate band. However, the $B(E 1) / B(E 2)$ ratios are difficult to extract for the low-lying levels where the low energy $E 2$ transitions are highly converted. On the other hand, low transition energies imply relatively long level lifetimes that can be directly measured with the fast timing $\beta \gamma \gamma(\mathrm{t})$ method $[3,4,5]$.

A recent introduction of the fast timing $\beta \gamma \gamma(\mathrm{t})$ method [6] at the PSB ISOLDE mass separator at CERN has opened the actinide region to lifetime measurements in the low picosecond range. Our specific interest is to examine three different aspects of the $B(E 1)$ systematics in the heavy Ra, Ac and Th nuclei related to the lowest-lying states that are the most representative of the ground-state collectivity: a) to map the nuclei which exhibit the effect of ' $B(E 1)$ quenching' - a sudden lowering of the $B(E 1)$ strength exhibited by specific nuclei in this region, b) to verify the $B(E 1)$ systematics at low excitations and to compare it with the better established $B(E 1)$ strength at higher spin and excitation energies - the latter seems to be higher because of the stabilizing effect of rotation, and c) to verify the predicted dependence of the $B(E 1)$ strength on specific intrinsic configurations of the parity-doublet (PD) bands in the odd nuclei. Furthermore, since our preliminary results indicate serious discrepancies with some of the previously quoted $D_{0}$ values (e.g. for ${ }^{222} \mathrm{Ra}$ and ${ }^{228} \mathrm{Ra}[2]$ ), there is a need not only to expand the systematics but also to verify (via our direct method of measurement) the results which were deduced by indirect methods.

In the present work we report the lifetime measurements for the octupole transitional ${ }^{227}$ Ra nucleus [7]. The measurements were performed at the PSB ISOLDE at CERN and combined with precise time calibrations of the fast timing detectors at the OSIRIS fissionproduct mass separator in Studsvik. Levels in ${ }^{227} \mathrm{Ra}$ were populated in the $\beta^{-}$decay of ${ }^{227} \mathrm{Fr}$. We note that the present results are independent of those obtained in our earlier run and reported in Ref. [6]. 
From the theoretical point of view, the octupole correlations in the actinide region [8] can be treated by strong coupling theories based on the assumption of a stable octupole deformation (see for example $[9,10]$ ). In these theories the octupole correlations are so strong as to lead to the formation of non-zero octupole (and induced dipole) moment in the mean field ground state. However, self-consistent Hartree-Fock or Hartree-FockBogoliubov calculations using the Skyrme [11] or Gogny [1, 12] effective nucleon-nucleon force, as well as Strutinsky method calculations [2], show only a few nuclei with stable octupole deformation of the ground state. The rest of the nuclei in the octupole collective actinide region are in fact only 'soft' with respect to octupole vibrations.

The second group of theoretical descriptions of octupole correlations (which seem well suited to the case of ${ }^{227} \mathrm{Ra}$ ) are the weak coupling theories, which involve all octupole correlations as residual interactions. In this case the average field of a nucleus remains reflection symmetric. However, relatively strong octupole correlations lead to highly collective octupole vibrational components in the low-lying states $[13,14,15,16]$. In this paper we demonstrate, in the examples of ${ }^{223} \mathrm{Ra}$ and ${ }^{227} \mathrm{Ra}$, that these octupole components cause intense dipole transitions between low-lying states and that the structure of these octupole components allows identification of the doublet character of the low-lying spectrum in a similar way to the strong coupling scheme with a stable octupole deformation. Furthermore, this work represents the first application of the weak coupling theory described in Section 5 to the calculation of transition rates in the actinide region, and thus provides a sensitive test of this approach.

Details of the measurements and data analysis for ${ }^{227} \mathrm{Ra}$ are discussed in Sections 2 and 3, and a comparison of the experimental data for the octupole transitional ${ }^{227} \mathrm{Ra}$ and the octupole deformed ${ }^{223} \mathrm{Ra}[7,17]$ is presented in Section 4 . A description of the theoretical model is given in Section 5, while the theoretical interpretation of the results for ${ }^{227} \mathrm{Ra}$ and ${ }^{223} \mathrm{Ra}$ is provided in Sections 6 and 7, respectively.

\section{$2 \quad$ Experimental details}

The measurements on ${ }^{227} \mathrm{Ra}$ were performed at the PSB ISOLDE mass separator facility at CERN. A highly pure beam of ${ }^{227} \mathrm{Rn}$ was produced from a $\mathrm{ThC}_{2}$ target (coupled to a plasma ion-source via a cold transfer line) bombarded with a $1 \mathrm{GeV}$ proton beam from the PS Booster. The beam was deposited on a movable magnetic tape and data were collected in two modes: a) when the tape was stationary, giving equal weights to the transitions from the decay of ${ }^{227} \mathrm{Rn}$ to ${ }^{227} \mathrm{Fr}\left(\mathrm{T}_{1 / 2}=2.5 \mathrm{~m}\right)$ and from ${ }^{227} \mathrm{Fr}$ to ${ }^{227} \mathrm{Ra}$ $\left(\mathrm{T}_{1 / 2}=42 \mathrm{~m}\right)$, and $\left.\mathrm{b}\right)$ when the tape was moving in a short cycle designed to suppress the observed intensity of transitions from the decay of ${ }^{227} \mathrm{Fr}$ to ${ }^{227} \mathrm{Ra}$. Here the results on the decay of ${ }^{227} \mathrm{Fr}$ to ${ }^{227} \mathrm{Ra}$ are reported, while the results on the decay of ${ }^{227} \mathrm{Rn}$ to ${ }^{227} \mathrm{Fr}$ are published separately [18].

The experimental set-up shown in Figure 1 consisted of four detectors positioned around the beam deposition point outside the experimental chamber (details of the chamber are not shown in the figure). The fast timing $\beta$-detector was a 3 -mm-thick NE111A plastic scintillator positioned behind the tape and separated from it by a thin $\mathrm{Al}$ window in the wall of the experimental chamber. There were also three $\gamma$-ray detectors: a small $\mathrm{BaF}_{2}$ crystal for fast timing and two Ge detectors with efficiencies of $40 \%$ and $70 \%$. A simple three-parameter time-delayed coincidence system was set between the $\beta$-detector and each of the $\gamma$-detectors. The data acquisition was performed in such a way that a valid event required a coincidence between the $\beta$-detector and any number of $\gamma$-detectors. Up to seven parameters were collected per coincident event: the energy of the $\beta$-particle, 
and two parameters (the energy of a $\gamma$-ray and the time-delay between the $\beta$-ray and this $\gamma$-ray) for each coincident $\gamma$-detector.

In the off-line analysis these coincident events were presorted into separate blocks of data corresponding to four independent measurements: two $\beta \gamma(\mathrm{t})$ recordings involving $\beta-\mathrm{Ge}$ or $\beta-\mathrm{BaF}_{2}$ detectors, and two $\beta \gamma \gamma(\mathrm{t})$ measurements involving $\beta-\mathrm{Ge}-\mathrm{Ge}$ or $\beta-\mathrm{BaF}_{2}-$ Ge detectors. From the first data set we obtained $\beta$-gated singles for each of the Ge detectors, and also found, from the time delay between events in the $\beta$-detector and a Ge detector, that none of the strongly populated states in ${ }^{227} \mathrm{Ra}$ has a lifetime in the range from 0.1 to $8 \mu \mathrm{s}$. From the time-delayed $\beta \gamma(t)$ events between fast timing detectors (the second data set) the lifetime of the $676-\mathrm{keV}$ level has been estimated. A partial level scheme of ${ }^{227} \mathrm{Ra}$ is given in Fig. 2.

\section{$2.1 \beta \gamma \gamma(\mathrm{t})$ Method}

Level lifetimes were measured with the $\beta \gamma \gamma(t)$ method (detailed in Refs. [3, 4, 5]), which involves triple coincidence $\beta-\mathrm{BaF}_{2}-\mathrm{Ge}$ events. The crucial timing information was obtained from the coincidences between the fast timing $\beta$ - and $\gamma$-detectors, while the coincidence with one of the Ge detectors served to select the desired decay branch.

Lifetimes with $T_{1 / 2} \geq 100 \mathrm{ps}$ were deconvoluted from the slope on the 'delayed' side of the time distribution, while shorter lifetimes were obtained from centroid shift measurements in the following way: When the level of interest is directly fed by $\beta$-decay, the mean life $\tau\left(\tau=T_{1 / 2} / \ln 2\right)$ is simply given by the difference between the centroid of the delayed time spectrum and the prompt centroid of the same $E_{\gamma}$. For indirect $\gamma$-ray feeding, the mean lifetime of interest is the difference between the centroid shift of the spectrum gated by the de-exciting $\gamma$-ray and the centroid shift of the spectrum gated by the feeding $\gamma$-transition. The time calibration of the $\mathrm{BaF}_{2}$ detector was obtained off-line at ISOLDE using a precalibrated source of ${ }^{140} \mathrm{Ba} \rightarrow{ }^{140} \mathrm{La} \rightarrow{ }^{140} \mathrm{Ce}$ that was prepared at the OSIRIS separator at Studsvik[20, 21]. Further cross-check and time-calibration was obtained on-site and in-beam from transitions in ${ }^{227} \mathrm{Rn} \rightarrow{ }^{227} \mathrm{Fr}$ and ${ }^{227} \mathrm{Fr} \rightarrow{ }^{227} \mathrm{Ra}$, which were analysed simultaneously by both the slope and centroid shift methods. More detailed time calibration of the full energy peak and Compton curves was done at OSIRIS using a variety of beams while maintaining the experimental geometry as close as possible to that at ISOLDE.

\section{Experimental results}

\section{1 $\gamma \gamma$-coincidences}

Using the $\gamma \gamma$-coincidences from the $\beta-\mathrm{Ge}-\mathrm{Ge}$ measurement we have verified the decay scheme of ${ }^{227} \mathrm{Fr} \rightarrow{ }^{227} \mathrm{Ra}$ previously proposed $[22,23]$ and identified the contaminating transitions, including the overlapping $\gamma$-transitions from the ${ }^{227} \mathrm{Rn} \rightarrow{ }^{227} \mathrm{Fr}$ decay. We have also extracted the previously unknown intensities of two low-energy transitions. These intensities, and upper limits for the intensities of a number of other transitions also needed for the evaluation of transition rates in Section 4, are listed in Table 1. Note that Table 1 also includes the results obtained with the Tardis multi-detector array in the measurements discussed in Ref. [18].

Furthermore, these data provided interpretation of the fast timing $\mathrm{BaF}_{2}$ spectra. $\mathrm{By}$ gating on a given $\gamma$-transition in one of the Ge detectors, the composition of the coincident $\mathrm{BaF}_{2}$ spectrum of poor energy resolution (from the $\beta-\mathrm{BaF}_{2}-\mathrm{Ge}$ experiment) is given by the equivalent high-resolution coincident Ge spectrum from the $\beta-\mathrm{Ge}-\mathrm{Ge}$ experiment. 


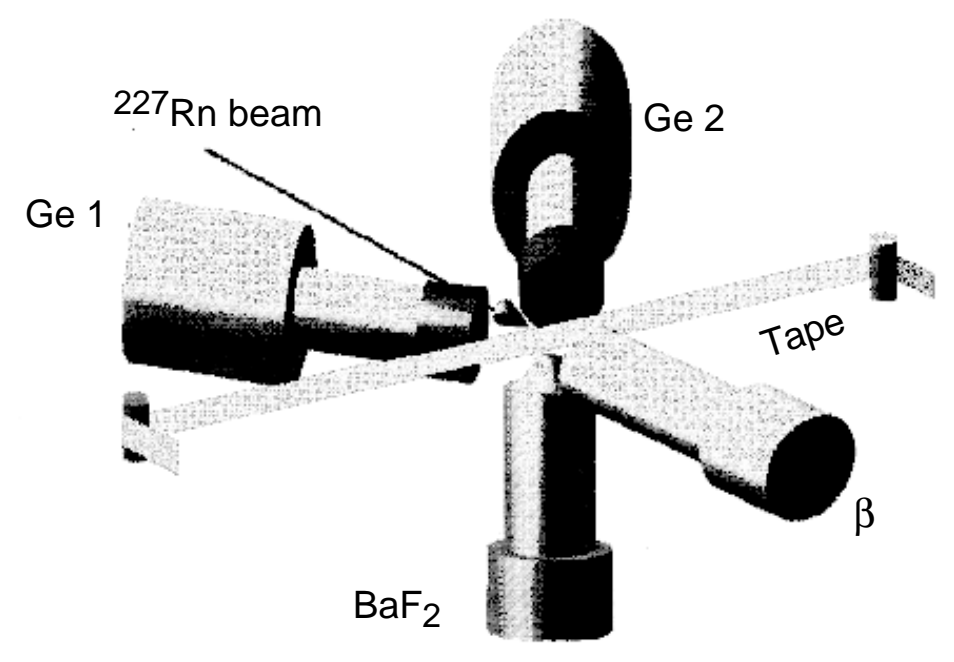

Figure 1: Schematic view of the experimental set-up with four detectors in a close geometry: the fast timing $\beta$ - and $\gamma$-detectors (a thin NE111A scintillator and a small $\mathrm{BaF}_{2}$ crystal) and two Ge detectors.

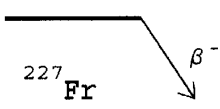

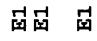

$\mathrm{T}_{1 / 2}$

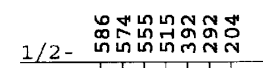
676

$<10 \mathrm{ps}$

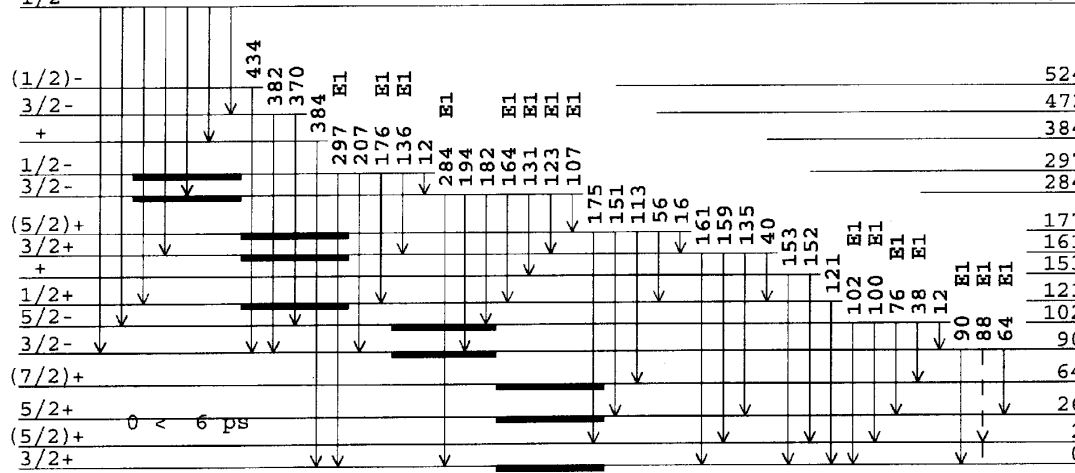

$<20 \mathrm{ps}$

$<21 \mathrm{ps}$

$<41$ ps

$<\begin{aligned} & <5 \\ & <39\end{aligned}$

$<47 \mathrm{ps}$

$236(30)$ ps

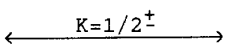

$\mathrm{K}=3 / 2 \pm$

${ }^{227} \mathrm{Ra}$

Figure 2: Partial decay scheme of ${ }^{227} \mathrm{Fr} \rightarrow{ }^{227} \mathrm{Ra}$ (from Ref. [19]) which includes transitions discussed in the text. Members of the $K^{\pi}=1 / 2^{ \pm}$and $3 / 2^{ \pm}$parity doublet bands are marked by thick lines, while the low-lying $E 1$ transitions are also indicated. The level half-lives are from this work. 
Table 1

Measured and estimated branching ratios for the unobserved transitions in ${ }^{227}$ Ra.

\begin{tabular}{|c|c|c|c|c|c|c|}
\hline \multirow[t]{2}{*}{$\mathrm{E}_{\gamma}$} & \multirow[t]{2}{*}{$\mathrm{E}_{\text {level }}$} & \multirow[t]{3}{*}{ Multipolarity } & \multicolumn{4}{|c|}{ Branching Ratio $^{a)}$} \\
\hline & & & \multicolumn{3}{|c|}{ Measured $^{\mathrm{b})}$} & Estimated ${ }^{\mathrm{c})}$ \\
\hline$(\mathrm{keV})$ & $(\mathrm{keV})$ & & This work & Tardis $\left.^{\mathrm{d}}\right)$ & Adopted & \\
\hline 11.9 & 101.9 & $\mathrm{E} 2(+\mathrm{M} 1)$ & $0.15(4)$ & $0.22(5)$ & $0.18(3)$ & $\sim 0.20$ \\
\hline 12.3 & 296.6 & $\mathrm{E} 2(+\mathrm{M} 1)$ & & $\leq 0.20$ & & $\sim 0.045$ \\
\hline 15.9 & 177.0 & $\mathrm{M} 1+\mathrm{E} 2$ & $\leq 0.45$ & $\leq 0.30$ & & $\sim 0.02$ \\
\hline 40.3 & 161.1 & $\mathrm{E} 2(+\mathrm{M} 1)$ & $\leq 0.17$ & $0.21(7)$ & $0.16(6)$ & $\sim 0.074$ \\
\hline 56.3 & 177.0 & E2 & $\leq 0.25$ & $\leq 0.30$ & & $\sim 0.11$ \\
\hline 88.3 & 90.0 & E1 & $\leq 0.06$ & $\leq 0.02$ & $\leq 0.02$ & \\
\hline 675.9 & 675.9 & E1 & $\leq 0.008^{\mathrm{e})}$ & & $\leq 0.008$ & \\
\hline
\end{tabular}

a) Ratio of the total transition intensity for the $\gamma$-ray listed in column 1 to the sum of the total intensities for all transitions de-exciting the same level (listed in column 2).

b) From $\gamma \gamma$; measured from the intensity of the $\gamma$-transitions cascading via the unobserved (highly converted) transition listed in column 1, except for the 88.3- and 675.9-keV transitions.

c) Using the lifetimes given in Table 2 and assuming $Q_{0} \sim 740(30)$.

d) Measured in a Compton-suppressed array, see Ref. [18].

e) From $\mathrm{I}_{\gamma}^{676 \mathrm{keV}} / \mathrm{I}_{\gamma}^{585 \mathrm{keV}}$; measured as $\leq 0.014$ in the $\beta$-gated $\gamma$-singles spectrum.

\subsection{Fast timing $\beta \gamma \gamma(t)$}

The lifetimes for the 90 - and 102-keV levels have been measured by the deconvolution method [3]. The $90-\mathrm{keV}$ level is intensely populated by the $586-\mathrm{keV}$ transition de-exciting the 676-keV level (see the partial decay scheme in Fig. 3b). In the triple coincidences between the $\beta, \mathrm{BaF}_{2}$ and Ge detectors, the $90-\mathrm{keV}$ transition selected in the Ge detector gives a coincident $\mathrm{BaF}_{2}$ spectrum, shown in Fig. 3a. With an additional gate on the full energy peak at $586 \mathrm{keV}$ in the $\mathrm{BaF}_{2}$ spectrum, the semi-prompt fast timing spectrum (Fig. $3 \mathrm{~b}$ ) between the $\beta$-rays and the $586-\mathrm{keV}$ transition is obtained. This spectrum indicates that the lifetime of the $676-\mathrm{keV}$ level is short (the slope on the time-delayed side gives a limit of $T_{1 / 2} \leq 30 \mathrm{ps}$ for the $676-\mathrm{keV}$ level: a result improved to $T_{1 / 2} \leq 10$ ps by a centroid shift measurement, see Table 2). By reversing the gates and selecting the $586-\mathrm{keV}$ transition in Ge and the 64/90 keV gate in $\mathrm{BaF}_{2}$ (see Fig. 3c), the time-delayed spectrum with a well-defined slope due to the lifetime of the $90-\mathrm{keV}$ level (Fig. 3d) is found. The slope fitting gives $T_{1 / 2}=254(9)$ ps which confirms the independent measurement of this level lifetime in our previous short run as $T_{1 / 2}=262(50)$ ps [6]. 
Table 2

Half-lives of the excited states in ${ }^{227} \mathrm{Ra}$.

\begin{tabular}{|rcl|}
\hline $\begin{array}{r}\text { Level } \\
(\mathrm{keV})\end{array}$ & $\begin{array}{c}T_{1 / 2} \\
\text { previous work } \\
(\mathrm{ps})\end{array}$ & $\begin{array}{c}T_{1 / 2} \\
\text { present work } \\
(\mathrm{ps})\end{array}$ \\
\hline 90.0 & $262(50) \mathrm{ps}$ & $254(9)$ \\
101.9 & & $236(30)$ \\
120.7 & & $\leq 47$ \\
161.1 & & $\leq 39$ \\
177.0 & & $\leq 58$ \\
284.3 & & $16(13) \mathrm{b})$ \\
296.6 & & $\leq 41$ \\
384.4 & & $\leq 21$ \\
471.6 & & $\leq 6$ \\
523.9 & & $\leq 20$ \\
675.9 & & $\leq 10{ }^{\mathrm{c})}$ \\
\hline
\end{tabular}

a) From Ref. [6].

b) Adopted value $T_{1 / 2} \leq 29 \mathrm{ps}$.

c) Estimated from the relative centroid shift of the $586-\mathrm{keV}$ peak in the fast timing $\beta-\mathrm{BaF}_{2}$ coincidences.
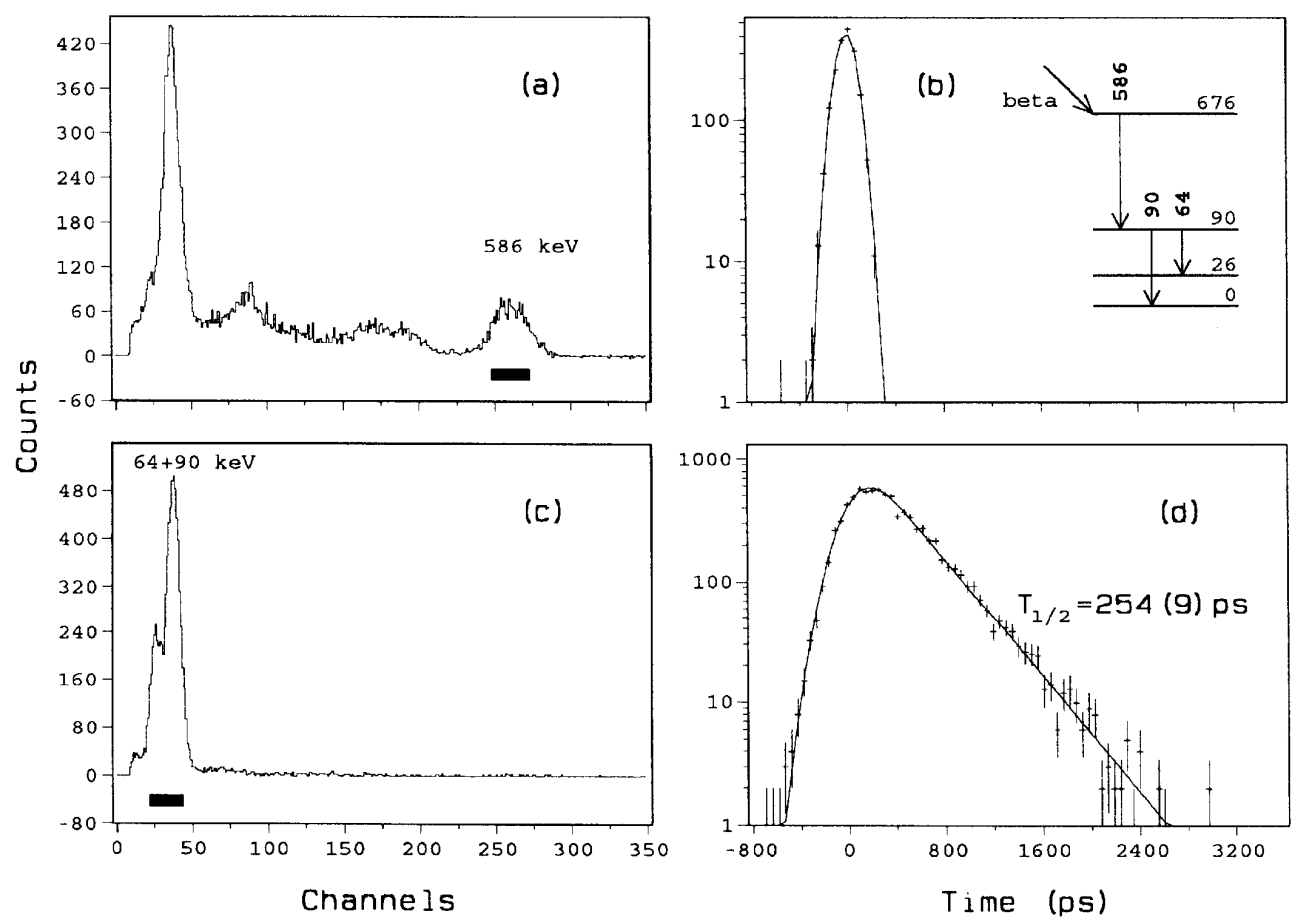

Figure 3: (a),(c): $\mathrm{BaF}_{2}$ spectra in coincidence with the 90 - and 586-keV transitions selected in $\mathrm{Ge}$, respectively. (b),(d): Fast timing $\left(\beta-\mathrm{BaF}_{2}\right)$ spectra gated on the full energy peak at $586 \mathrm{keV}$ in spectrum (a), and on the $64 / 90-\mathrm{keV}$ peak in spectrum (c), respectively. The gated regions are marked by black rectangles in spectra (a) and (c). 
The timing analysis for the $102-\mathrm{keV}$ level is affected by the highly converted and fairly intense (15\%) 12-keV transition between the 102- and 90-keV levels (see Fig. 4 and Table 1). This introduces an extra time-delayed component into the time spectrum of Fig. 4 because of the $90-\mathrm{keV}$ transition de-exciting the $90-\mathrm{keV}$ level that got mixed into the $\mathrm{BaF}_{2}$ gate as an impurity. We estimate its contribution to be below $8 \%$. The time distribution analysis does not indicate any significant presence of a second time component, implying perhaps a smaller impurity contribution than estimated. Nevertheless, the uncertainty in the lifetime of the 102-keV level has been judiciously increased.
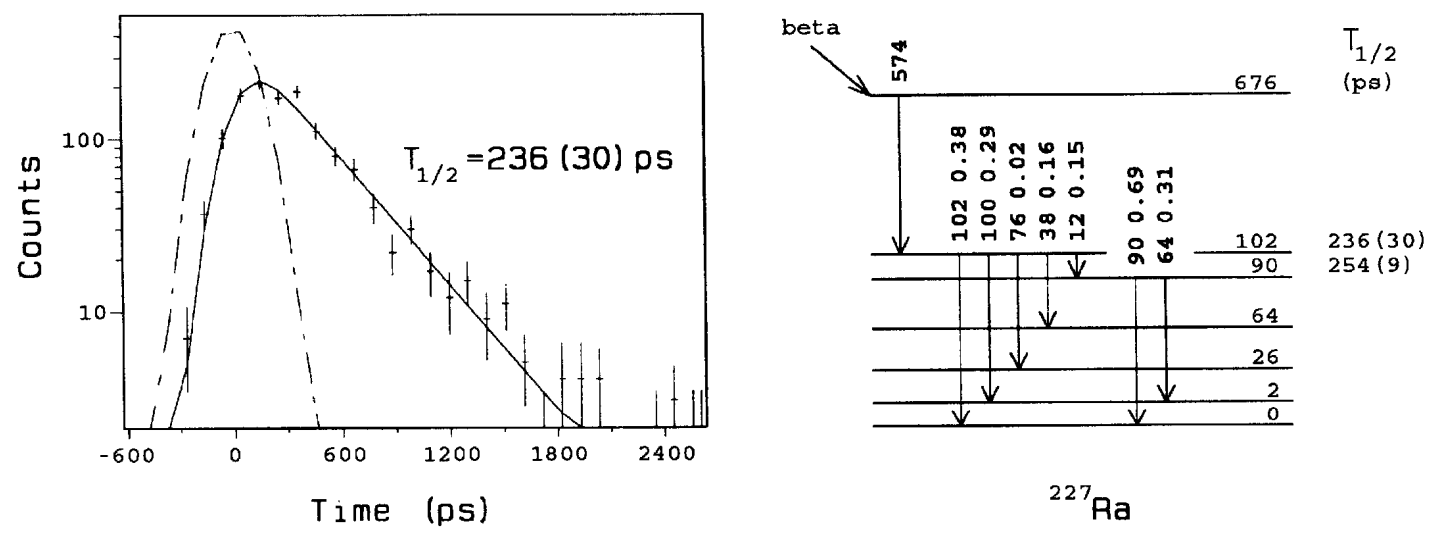

Figure 4: Left: Time-delayed $\left(\beta-\mathrm{BaF}_{2}\right)$ spectrum gated by the $\gamma$-energy window from 85 to $115 \mathrm{keV}$ in the $\mathrm{BaF}_{2}$ spectrum and by the $574-\mathrm{keV}$ transition in the Ge detector. The slope is due to the lifetime of the $102-\mathrm{keV}$ level. The uncertainty in $T_{1 / 2}$ was increased to account for the $15 \%$ branching to the $90-\mathrm{keV}$ level and additional time delay caused by a partial presence of the $90-\mathrm{keV}$ transition in the $\mathrm{BaF}_{2}$ gate. Right: A partial decay scheme of ${ }^{227} \mathrm{Fr} \rightarrow{ }^{227} \mathrm{Ra}$.

Shorter lifetimes were measured by the centroid shift technique (for details including corrections applied see Refs. [3, 5]). Since the prompt and delayed time spectra must be measured concurrently in order to maintain identical experimental conditions, the time calibration involves either the 'prompt points' or the 'reference points', which are internal to the decay and serve to renormalize the relative prompt curve for the $\mathrm{BaF}_{2}$ detector that was measured off-line. Unlike the prompt points, for which by definition $\tau=0$, the reference points may be shifted by a non-zero time delay (due to the lifetime of the level they de-excite), which exactly cancels out in the timing analysis and thus becomes irrelevant (see also Ref. [24]).

The 204-, 292-, 392- and 586-keV transitions de-exciting the $676-\mathrm{keV}$ state were selected as the reference points. [Conveniently, this level is strongly $\beta$-fed $(52 \%)$ and deexcites by a few relatively-strong two $\gamma$-ray cascades, as seen in the partial decay scheme in Fig. 5.] The centroids of the time spectra gated by the feeding $\beta$-ray and the full energy peaks of these transitions selected in $\mathrm{BaF}_{2}$ were obtained, using two $\gamma$-ray cascades, by selecting in the Ge detector the bottom member of the cascade. As an example, consider the $586-90-\mathrm{keV}$ cascade in Fig. 3: the centroid of the time spectrum shown in Fig. 3b provides the reference point at $586 \mathrm{keV}$. 

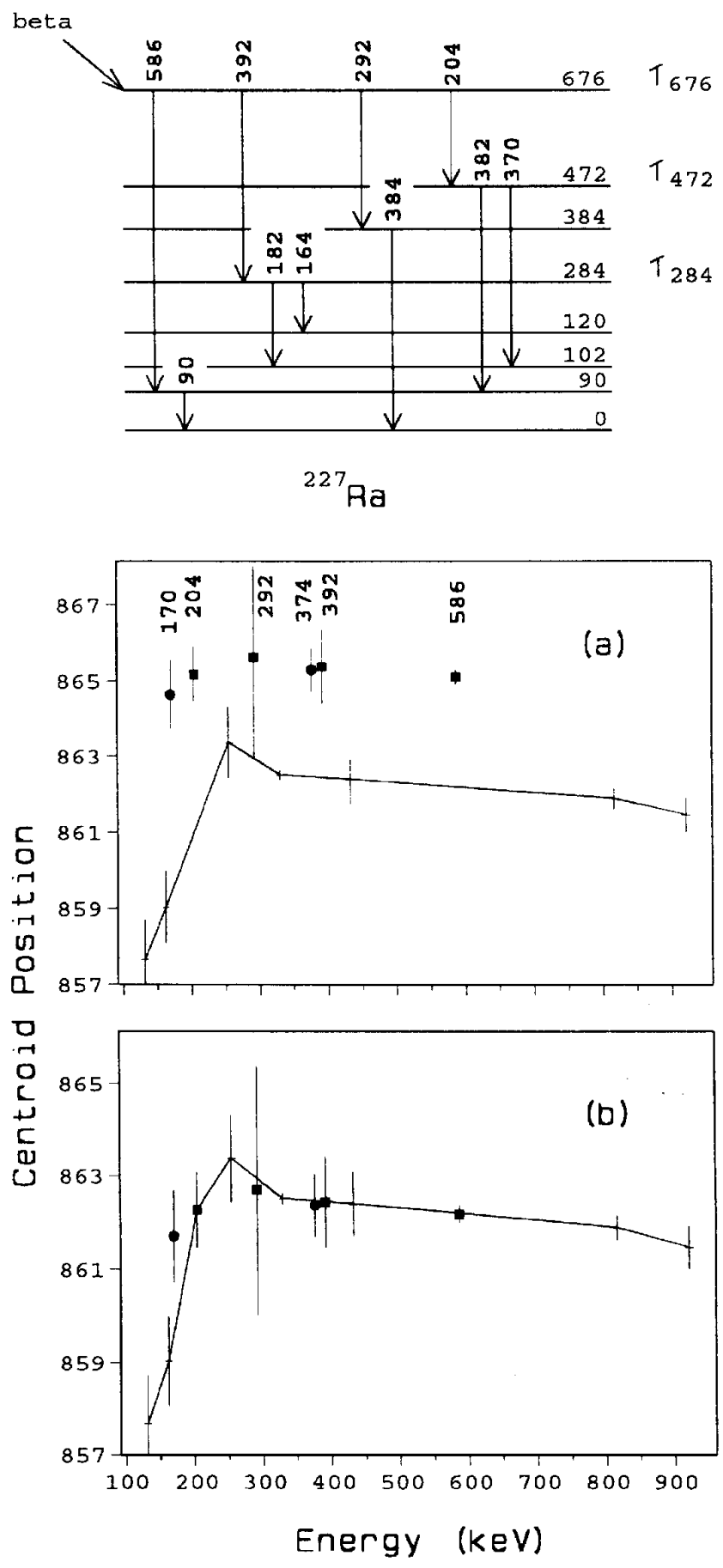

Figure 5: Top: A partial decay scheme of ${ }^{227} \mathrm{Fr} \rightarrow{ }^{227}$ Ra showing a few double $\gamma$-ray cascades deexciting the $676-\mathrm{keV}$ level. Middle: Centroid positions of the fast timing $\left(\beta-\mathrm{BaF}_{2}\right)$ spectra for a few $\gamma$-transitions measured off-line and in-beam: the prompt curve (solid lines) connects prompt points that were measured off-line using the $A=140$ source. The solid squares indicate time centroids for the transitions deexciting directly the $676-\mathrm{keV}$ level, which define the internal reference points. Bottom: The centroid positions of the ${ }^{227} \mathrm{Ra}$ points after shifting down by a constant (see text for details). The calibration of the vertical scale is 13.1 ps per channel. 
Figure 5a illustrates some of the in-beam data for ${ }^{227} \mathrm{Ra}$ (solid symbols) and the relative prompt curve measured off-line with the $A=140$ source (crosses connected by lines). The reference points (solid squares marked by the transition energy in keV: 204, 292, 392, and 586) are shifted from the true prompt positions by the mean life of the $676-\mathrm{keV}$ state, $\tau_{676}$, the exact value of which remains unknown. The solid dot ' 374 ' marks the centroid of the time spectrum gated by the 204-keV transition in Ge and a common gate at $374 \mathrm{keV}$ in $\mathrm{BaF}_{2}$ (on the unresolved full energy peaks at 370 and $382 \mathrm{keV}$ ), that is shifted from the true prompt by the sum of the mean lives for the 676- and 472-keV levels: $\tau_{676}+\tau_{472}$. Similarly, the solid dot ' 170 ' marks the centroid of the time spectrum gated by the $392-\mathrm{keV}$ transition in $\mathrm{Ge}$ and a common gate at $170 \mathrm{keV}$ in $\mathrm{BaF}_{2}$ (on the unresolved full energy peaks at 164 and $182 \mathrm{keV}$ ). Its shift from the true prompt is the sum of the mean lives: $\tau_{676}+\tau_{284}$. It can be easily seen that the relative shifts of the ' 170 ' and ' 374 ' centroids from the reference points directly give the mean lives for the 284- and 472-keV levels, respectively, provided the exact shape of the curve connecting the reference points and its shape below the energy of $204 \mathrm{keV}$ are known.

This shape is provided by the relative prompt curve. In Fig. 5b, the in-beam points were moved down by a constant selected in such a way as to make the reference points at 292, 392, and $586 \mathrm{keV}$ overlap with the relative prompt curve. (The point at $204 \mathrm{keV}$ was used after the renormalization to redefine the shape of the relative prompt curve at $204 \mathrm{keV}$.) One can now provide the numerical estimates for the centroid shifts. In particular, the shift of the ' 374 ' point relative to the reference points is negligible indicating a short lifetime for the $472-\mathrm{keV}$ level (the measured value is $T_{1 / 2} \leq 6 \mathrm{ps}$ ), while the ' 170 ' point is significantly shifted, and the related uncertainties are quite large as well. The measured shift of $1.2 \pm 1.0$ channels (see Fig. 5b) yields $T_{1 / 2}=16 \pm 13$ ps. In general, a few independent measurements were made for each level and the final half-life results, listed in Table 2, represent the weighted average.

\section{$4 \quad$ Systematics of the $B(E \mathbf{1})$ rates in ${ }^{223} \mathbf{R a}$ and ${ }^{227} \mathbf{R a}$}

The occurence of stable octupole deformation is manifested in the odd-A nuclei by a few characteristic fingerprints such as degenerate parity doublet (PD) bands, equal magnetic moments for the parity doublets, enhanced $E 1$ transitions between members of the PD band, and decoupling parameters for the $K=1 / 2$ bands with equal values but opposite signs [25]. In order to compare these predictions with experimental values, Fig. 6 shows the energy systematics of the $K^{\pi}=1 / 2^{ \pm}$and $K^{\pi}=3 / 2^{ \pm}$PD bands, while Table 3 shows the systematics of the decoupling parameters measured for the $K^{\pi}=1 / 2^{ \pm}$bands in ${ }^{223,225,227} \mathrm{Ra}$. Clearly these predictions are only partially fulfilled in ${ }^{223,225,227} \mathrm{Ra}$. Although the smooth energy systematics and moderate variations in the decoupling parameters do not suggest strong differences in the structure of these nuclei (indeed it has been shown that octupole collectivities must be involved in all three nuclei), it is the degree and kind of octupole collectivity that set these nuclei apart with particularly different interpretation of ${ }^{223} \mathrm{Ra}$ and ${ }^{227} \mathrm{Ra}$. Consequently, the intercomparison of the latter two nuclei will be the focal point of the remainder of this paper, since the degree and the kind of octupole collectivity for the heavier Ra nuclei remain an open question. 
Table 3

The experimental decoupling parameters for the $K^{\pi}=1 / 2^{ \pm}$parity-doublet bands in ${ }^{223,225,227} \mathrm{Ra}$, and the calculated results (this work) for ${ }^{223,227} \mathrm{Ra}$.

\begin{tabular}{|c|ccccc|}
\hline \multirow{2}{*}{} & \multicolumn{5}{|c|}{ Decoupling parameters } \\
\cline { 2 - 6 } & \multicolumn{2}{|c}{${ }^{223} \mathrm{Ra}^{\mathrm{a})}$} & ${ }^{225} \mathrm{Ra}^{\mathrm{b})}$ & ${ }^{227} \mathrm{Ra}^{\mathrm{a})}$ \\
& Exp. & Theor. & Exp. & Exp. & Theor. \\
\hline$K^{\pi}=1 / 2^{+}$ & 1.35 & 1.21 & 1.53 & 0.62 & 1.17 \\
$K^{\pi}=1 / 2^{-}$ & -2.0 & -2.48 & -2.59 & -1.68 & -2.37 \\
\hline
\end{tabular}

a) Taken from [19].

b) Taken from [26].

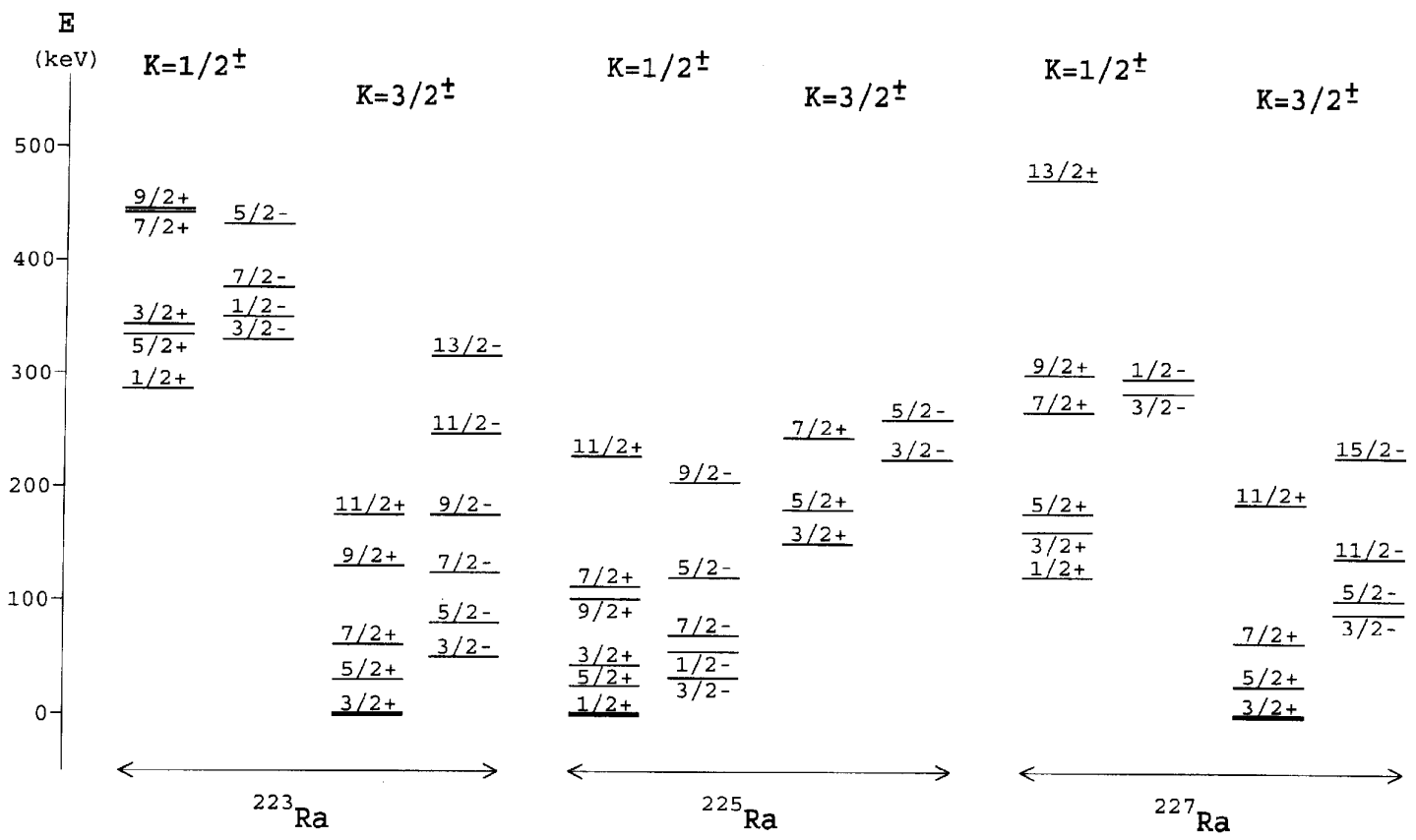

Figure 6: Systematics of the $K=1 / 2^{ \pm}$and $K=3 / 2^{ \pm}$parity doublet bands in ${ }^{223,225,227} \operatorname{Ra}$ (from Refs. [19, 25]). Note that these bands remain at the low excitation energy with a small and almost constant energy separation between the $K^{\pi}=3 / 2^{-}$and $3 / 2^{+}$partner bands. Small separation energy also characterizes the $K^{\pi}=1 / 2^{ \pm}$partners in ${ }^{223,225} \operatorname{Ra}$ with a significantly wider gap in ${ }^{227} \mathrm{Ra}$.

Prior to this work the nuclear structures of ${ }^{223} \mathrm{Ra}$ and ${ }^{227} \mathrm{Ra}$ were analysed with the models of strong and of intermediate coupling between the particle and the reflectionasymmetric intrinsic field [8], the non-adiabatic rigid reflection-asymmetric rotor model $[9,17]$, the Strutinski shell-correction method with a single-particle level scheme obtained with a Woods-Saxon mean field [27], or more generally, using the reflection-asymmetric mean field approach with an average Woods-Saxon potential and a monopole-pairing residual interaction [7]. Although the height of the octupole barrier in ${ }^{223} \mathrm{Ra}$ remains sensitive to the details of the models [7, 8, 27], there is a consensus in these studies that ${ }^{223} \mathrm{Ra}$ is a nucleus with stable octupole deformation $\left(\epsilon_{3}=0.10\right)$. There is also a consensus that ${ }^{227} \mathrm{Ra}$ lies on the border of the octupole-instability region. It is thus an octupole transitional nucleus with a possible small and stable octupole deformation 
$\left(\epsilon_{3} \approx 0.07\right)$ and with some evidence for shape coexistence of pure quadrupole and quadrupole-octupole deformations [19]; or alternatively, ${ }^{227} \mathrm{Ra}$ with a shallow potential well for $\beta_{3}$ may be considered as an octupole vibrational nucleus. It should be noted, however, that the amount of octupole correlations is state-dependent with particularly large fluctuations predicted at the border region [7].

\subsection{Fast intra-parity-doublet $E 1$ transitions}

The new experimental data allow another important characteristic of the octupole collectivity, namely fast $E 1$ intra-PD transitions, to be examined for the first time in ${ }^{227}$ Ra. Tables 4 and 5 provide a summary of the measured transition rates for ${ }^{227} \mathrm{Ra}$ and ${ }^{223} \mathrm{Ra}$, respectively, including the measured $B(E 1)$ rates, while Table 6 lists the measured and calculated $D_{0}$ values for the PD bands in ${ }^{223} \mathrm{Ra}$ and ${ }^{227} \mathrm{Ra}$. The dipole moment $D_{0}$ is defined via the rotational formula

$$
B\left(E 1 ; I_{i} \rightarrow I_{f}\right)=\frac{3}{4 \pi} D_{0}^{2}\left\langle I_{i} K_{i} 10 \mid I_{f} K_{f}\right\rangle^{2},
$$

which is not strictly applicable to the transitional nuclei. Nevertheless, $D_{0}$, treated here as a parameter, provides a consistent means for intercomparison of the $B(E 1)$ strength between even-even and odd-A nuclei as well as for the $E 1$ transitions de-exciting various band members. 
Table 4

Experimental and theoretical transition probabilities, $\gamma$-branching ratios, and decay half-lives in ${ }^{227}$ Ra. Experimental data from this work and Ref. [19].

\begin{tabular}{|c|c|c|c|c|c|c|c|c|c|c|}
\hline \multirow{3}{*}{$\begin{array}{c}\text { Initial } \\
\text { state } \\
(\mathrm{keV})\end{array}$} & \multirow{3}{*}{$\begin{array}{l}\text { Final } \\
\text { state } \\
(\mathrm{keV}) \\
\end{array}$} & \multirow[t]{3}{*}{$b_{i} b_{f}^{a)}$} & \multirow{3}{*}{$\begin{array}{c}\gamma \text {-ray } \\
E_{\gamma} \\
(\mathrm{keV})\end{array}$} & \multicolumn{3}{|c|}{$\left.B(X L ; i \rightarrow f)^{\mathrm{b}, \mathrm{c}}\right)$} & \multirow{2}{*}{\multicolumn{2}{|c|}{$\begin{array}{c}\gamma \text {-branching } \\
\text { ratio }^{\mathrm{d})}\end{array}$}} & \multirow{2}{*}{\multicolumn{2}{|c|}{$\begin{array}{l}T_{1 / 2}^{(i)} \\
(\mathrm{ps})\end{array}$}} \\
\hline & & & & & & & & & & \\
\hline & & & & $X L$ & Exp. ${ }^{\text {) }}$ & Theor. & Exp. & Theor. & Exp. & Theor. $^{c)}$ \\
\hline \multirow[t]{4}{*}{64.1} & 25.8 & $b b$ & 38.3 & M1 & & $1.1 \times 10^{-1}$ & & 855 & & 67 \\
\hline & & & & E2 & & $1.8 \times 10^{4}$ & & & & \\
\hline & 1.7 & $b c$ & 62.4 & M1 & & $2.8 \times 10^{-2}$ & & 926 & & \\
\hline & 0.0 & $b b$ & 64.1 & E2 & & $9.8 \times 10^{3}$ & 100 & 100 & & \\
\hline \multirow[t]{3}{*}{90.0} & 25.8 & $B b$ & 64.3 & E1 & $1.44(16) \times 10^{-3}$ & $1.2 \times 10^{-3}$ & $38(4)$ & 20 & $254(9)$ & 184 \\
\hline & 1.7 & $B c$ & 88.3 & E1 & $\leq 4.8 \times 10^{-5}$ & $1.1 \times 10^{-4}$ & $\leq 3.3$ & 4.7 & & \\
\hline & 0.0 & $B b$ & 90.0 & E1 & $1.41(8) \times 10^{-3}$ & $2.2 \times 10^{-3}$ & $100(10)$ & 100 & & \\
\hline \multirow[t]{6}{*}{101.9} & 90.0 & $B B$ & 11.9 & M1 & $\leq 4.9 \times 10^{-2 \mathrm{f})}$ & $1.4 \times 10^{-1}$ & $\leq 0.12$ & 0.22 & $236(30)$ & 143 \\
\hline & & & & $\mathrm{E} 2$ & $\left.\leq 2.5 \times 10^{4} \mathrm{~g}\right)$ & $2.7 \times 10^{4}$ & & & & \\
\hline & 64.1 & $B b$ & 37.9 & E1 & $2.1(5) \times 10^{-3}$ & $7.0 \times 10^{-4}$ & $18(4)$ & 3.3 & & \\
\hline & 25.8 & $B b$ & 76.1 & E1 & $5.9(13) \times 10^{-5}$ & $6.0 \times 10^{-5}$ & $4.2(7)$ & 2.3 & & \\
\hline & 1.7 & $B c$ & 100.1 & E1 & $4.7(10) \times 10^{-4}$ & $9.1 \times 10^{-5}$ & $76(16)$ & 7.8 & & \\
\hline & 0.0 & $B b$ & 101.9 & E1 & $5.8(10) \times 10^{-4}$ & $1.1 \times 10^{-3}$ & $100(16)$ & 100 & & \\
\hline \multirow[t]{4}{*}{120.7} & 90.0 & $a B$ & 30.7 & E1 & & $3.2 \times 10^{-3}$ & & 0.53 & $\leq 47$ & 2.4 \\
\hline & 25.8 & $a b$ & 95.0 & $\mathrm{E} 2$ & & $1.6 \times 10^{2}$ & & 0.006 & & \\
\hline & 1.7 & $a c$ & 118.8 & $\mathrm{E} 2$ & & $5.0 \times 10^{-2}$ & & $\sim 0$ & & \\
\hline & 0.0 & $a b$ & 120.7 & M1 & $\left.\geq 4.5 \times 10^{-2 h}\right)$ & $8.9 \times 10^{-1}$ & 100 & 100 & & \\
\hline \multirow[t]{7}{*}{161.1} & 120.7 & $a a$ & 40.3 & M1 & & $1.1 \times 10^{-1}$ & $\leq 5$ & 1.9 & $\leq 39$ & $\approx 5.2$ \\
\hline & & & & E2 & & $4.1 \times 10^{3}$ & & & & \\
\hline & 101.9 & $a B$ & 59.2 & E1 & & $2.0 \times 10^{-3}$ & & 10.1 & & \\
\hline & 90.0 & $a B$ & 71.1 & E1 & & $3.1 \times 10^{-3}$ & & 27 & & \\
\hline & 25.8 & $a b$ & 135.3 & M1 & $\left.\geq 2.2 \times 10^{-2 \mathrm{~h}}\right)$ & $1.5 \times 10^{-1}$ & $100(29)$ & 100 & & \\
\hline & 1.7 & $a c$ & 159.4 & M1 & $\left.\geq 3.9 \times 10^{-3 \mathrm{~h}}\right)$ & $6.6 \times 10^{-2}$ & $31(3)$ & 72 & & \\
\hline & 0.0 & $a b$ & 161.1 & M1 & $\left.\geq 7.4 \times 10^{-3 \mathrm{~h}}\right)$ & $1.1 \times 10^{-1}$ & $60(6)$ & 124 & & \\
\hline \multirow[t]{12}{*}{177.0} & 161.1 & $a a$ & 15.9 & M1 & & $2.0 \times 10^{-1}$ & $\leq 1.8^{\mathrm{i})}$ & 1.0 & $\leq 58$ & $\approx 19$ \\
\hline & & & & E2 & & $4.8 \times 10^{4}$ & & & & \\
\hline & 120.7 & $a a$ & 56.3 & E2 & & $5.6 \times 10^{3}$ & $\left.\leq 1.8^{\mathrm{i}}\right)$ & 0.27 & & \\
\hline & $(115)$ & $a B$ & $(62)$ & E1 & & $2.0 \times 10^{-4}$ & & 5.3 & & \\
\hline & 101.9 & $a B$ & 75.0 & $\mathrm{E} 1$ & & $1.1 \times 10^{-3}$ & & 52 & & \\
\hline & 90.0 & $a B$ & 86.9 & E1 & & $3.1 \times 10^{-4}$ & & 23 & & \\
\hline & 83.4 & $a c$ & 93.6 & E2 & & $3.1 \times 10^{0}$ & & $\sim 0$ & & \\
\hline & 64.1 & $a b$ & 113.0 & M1 & $\left.\geq 4.0 \times 10^{-3} \mathrm{~h}\right)$ & $1.8 \times 10^{-2}$ & $18(7)$ & 32 & & \\
\hline & $(35)$ & $a c$ & $(142)$ & M1 & & $1.5 \times 10^{-2}$ & & 53 & & \\
\hline & 25.8 & $a b$ & 151.2 & M1 & $\left.\geq 5.2 \times 10^{-3} \mathrm{~h}\right)$ & $2.5 \times 10^{-2}$ & $63(43)$ & 107 & & \\
\hline & 1.7 & $a c$ & 175.2 & M1 & $\left.\geq 5.8 \times 10^{-3 \mathrm{~h}}\right)$ & $1.5 \times 10^{-2}$ & $100(67)$ & 100 & & \\
\hline & 0.0 & $a b$ & 177.0 & M1 & & $1.4 \times 10^{-2}$ & & 96 & & \\
\hline \multirow[t]{12}{*}{284.3} & 177.0 & $A a$ & 107.3 & E1 & $\left.\sim 2.9 \times 10^{-3} \mathrm{j}\right)$ & $4.9 \times 10^{-3}$ & $38(4)$ & 42 & $16(13)$ & $\leq 12$ \\
\hline & 161.1 & $A a$ & 123.2 & E1 & $\left.\sim 4.4 \times 10^{-4} \mathrm{j}\right)$ & $3.5 \times 10^{-4}$ & $9(1)$ & 4.5 & & \\
\hline & 153.3 & $A ?$ & 131.0 & E1 & $\left.\sim 3.0 \times 10^{-4} \mathrm{j}\right)$ & & $7(1)$ & & & \\
\hline & 120.7 & $A a$ & 163.6 & $\mathrm{E} 1$ & $\left.\sim 2.2 \times 10^{-3} \mathrm{j}\right)$ & $3.3 \times 10^{-3}$ & $100(10)$ & 100 & & \\
\hline & (115) & $A B$ & (169) & $\mathrm{E} 2$ & & $2.7 \times 10^{3}$ & & 2.0 & & \\
\hline & 101.9 & $A B$ & 182.4 & M1 & $\left.\sim 1.6 \times 10^{-2} \mathrm{j}, \mathrm{k}\right)$ & $1.5 \times 10^{-2}$ & $17(2)$ & 9.3 & & \\
\hline & & & & $\mathrm{E} 2$ & $\left.\sim 3.5 \times 10^{3} \mathrm{j}, \mathrm{k}\right)$ & $2.1 \times 10^{3}$ & & & & \\
\hline & 90.0 & $A B$ & 194.3 & M1 & & $7.3 \times 10^{-3}$ & $10(1)$ & 6.9 & & \\
\hline & & & & $\mathrm{E} 2$ & & $1.9 \times 10^{3}$ & & & & \\
\hline & 25.8 & $A b$ & 258.3 & E1 & & $9.3 \times 10^{-7}$ & & 0.11 & & \\
\hline & 1.7 & $A c$ & 282.4 & E1 & & $2.6 \times 10^{-5}$ & & 4.1 & & \\
\hline & 0.0 & $A b$ & 284.3 & E1 & $\left.\sim 4.0 \times 10^{-5} \mathrm{j}\right)$ & $5.6 \times 10^{-5}$ & $10(2)$ & 8.9 & & \\
\hline
\end{tabular}


Table 4 cont.

\begin{tabular}{|ccccccccccc|}
\hline 296.6 & 284.3 & $A A$ & 12.3 & M1 & & $1.8 \times 10^{-1}$ & $\left.\leq 0.19^{\mathrm{i}}\right)$ & 0.02 & $\leq 41$ & $\leq 9.5$ \\
& & & & $\mathrm{E} 2$ & & $3.2 \times 10^{4}$ & & & & \\
& 161.1 & $A a$ & 135.5 & $\mathrm{E} 1$ & $\geq 7.6 \times 10^{-4}$ & $5.7 \times 10^{-3}$ & $100(38)$ & 79 & & \\
& 120.7 & $A a$ & 175.9 & $\mathrm{E} 1$ & $\geq 3.8 \times 10^{-4}$ & $3.3 \times 10^{-3}$ & $100(23)$ & 100 & & \\
& 101.9 & $A B$ & 194.7 & $\mathrm{E} 2$ & & $1.2 \times 10^{3}$ & & 1.4 & & \\
& 90.0 & $A B$ & 206.6 & $\mathrm{M} 1$ & $\left.\geq 1.4 \times 10^{-2 \mathrm{f}}\right)$ & $1.6 \times 10^{-2}$ & $52(5)$ & 11.3 & & \\
& & & & $\mathrm{E} 2$ & $\left.\geq 4.8 \times 10^{3 \mathrm{~g}}\right)$ & $1.6 \times 10^{3}$ & & & & \\
& 0.0 & $A b$ & 296.6 & $\mathrm{E} 1$ & $\geq 1.5 \times 10^{-6}$ & $1.3 \times 10^{-5}$ & $3.1(15)$ & 1.9 & & \\
\hline 675.9 & 475.0 & $D ?$ & 200.9 & $\mathrm{E} 1$ & $\geq 5.5 \times 10^{-5}$ & & $2.0(2)$ & & $\leq 10$ & $\leq 0.84$ \\
& 471.6 & $D ?$ & 204.3 & $\mathrm{M} 1$ & $\geq 1.5 \times 10^{-2 \mathrm{~m})}$ & & $9.9(8)$ & & & \\
& 438.8 & $D ?$ & 237.2 & $\mathrm{E} 1$ & $\geq 2.5 \times 10^{-6}$ & & $0.19(5)$ & & & \\
& 384.4 & $D ?$ & 291.6 & $\mathrm{E} 1$ & $\geq 2.3 \times 10^{-5}$ & & $2.5(2)$ & & & \\
& 296.6 & $D A$ & 379.2 & $\mathrm{M} 1$ & $\geq 1.1 \times 10^{-4}$ & $2.5 \times 10^{-3}$ & $0.32(5)$ & 0.62 & & \\
& 284.3 & $D A$ & 391.6 & $\mathrm{M} 1$ & $\left.\geq 3.0 \times 10^{-3 \mathrm{~h}}\right)$ & $8.0 \times 10^{-2}$ & $8.8(6)$ & 22 & & \\
161.1 & $D a$ & 514.8 & $\mathrm{E} 1$ & $\geq 9.7 \times 10^{-6}$ & $3.0 \times 10^{-4}$ & $6(1)$ & 17 & & \\
& 120.7 & $D a$ & 555.2 & $\mathrm{E} 1$ & $\geq 1.4 \times 10^{-5}$ & $6.5 \times 10^{-4}$ & $10.1(7)$ & 45 & & \\
101.9 & $D B$ & 573.8 & $\mathrm{E} 2$ & $\geq 7.4$ & $2.8 \times 10^{1}$ & $1.6(1)$ & 0.54 & & \\
90.0 & $D B$ & 585.8 & $\mathrm{M} 1$ & $\geq 1.1 \times 10^{-2 \mathrm{~h})}$ & $1.1 \times 10^{-1}$ & $100(5)$ & 100 & & \\
0.0 & $D b$ & 675.9 & $\mathrm{E} 1$ & & $4.5 \times 10^{-5}$ & $\leq 1.4$ & 5.7 & & \\
\hline
\end{tabular}

a) Band assignment for the initial and final states: $a, b, c, A$, and $B$ stand for the $K^{\pi}=1 / 2^{+}, 3 / 2^{+}, 5 / 2^{+}, 1 / 2^{-}$, and $3 / 2^{-}$PD bands, respectively. $D$ and '?' stand for the $K^{\pi}=1 / 2^{-}$band at $675.9 \mathrm{keV}$ and for an unclassified level, respectively.

b) In units of $\mathrm{e}^{2} \cdot \mathrm{fm}^{2}$ for $E 1, \mathrm{e}^{2} \cdot \mathrm{fm}^{4}$ for $E 2$, and $\mu_{N}^{2}$ for $M 1$ transitions; see the $X L$ multipolarity in column 5 .

c) Total conversion coefficients were taken from Refs. [19, 28].

d) Relative to the strongest $\gamma$-transition intensity for a given level; $\gamma$-intensity combines the $M 1$ and $E 2$ components for the mixed $M 1 / E 2$ transitions.

e) Includes, as corrections for the 12.3-, 15.9- and 56.3-keV transitions, the estimates from column 7 in Table 1.

f) $\delta(E 2 / M 1)$ unknown, assuming $|\delta(E 2 / M 1)| \geq 0$.

g) $\delta(E 2 / M 1)$ unknown, assuming $|\delta(E 2 / M 1)| \leq \infty$.

h) $\delta(E 2 / M 1)$ unknown, assuming $|\delta(E 2 / M 1)| \sim 0$.

i) Using the measured branching ratios of $\leq 0.20, \leq 0.30$, and $\leq 25$ for the 12.3-, 15.9-, and 56.3-keV transitions, respectively, from Table 1.

j) Transition rates at $T_{1 / 2}=16 \mathrm{ps}$.

k) Using $\delta(E 2 / M 1)=0.7$, Ref. [19].

1) $\delta(E 2 / M 1)$ unknown, assuming $|\delta(E 2 / M 1)| \sim \infty$.

m) Using $\delta(E 2 / M 1) \leq 0.8$, Ref. [19]. 


\section{Table 5}

Experimental and theoretical transition probabilities, $\gamma$-branching ratios, and decay half-lives in ${ }^{223}$ Ra. Experimental data from Refs. [19, 27, 29].

\begin{tabular}{|c|c|c|c|c|c|c|c|c|c|c|}
\hline \multirow{3}{*}{$\begin{array}{c}\text { Initial } \\
\text { state } \\
(\mathrm{keV})\end{array}$} & \multirow{3}{*}{$\begin{array}{c}\text { Final } \\
\text { state } \\
(\mathrm{keV})\end{array}$} & \multirow{3}{*}{$\begin{array}{c}\left.b_{i} b_{f}{ }^{\mathrm{a}}\right) \\
(\mathrm{keV})\end{array}$} & \multirow{3}{*}{$\begin{array}{c}\gamma \text {-ray } \\
E_{\gamma}\end{array}$} & \multicolumn{3}{|c|}{$B(X L ; i \rightarrow f)^{\mathrm{b}, \mathrm{c})}$} & \multirow{2}{*}{\multicolumn{2}{|c|}{$\begin{array}{c}\gamma \text {-branching } \\
\text { ratio }^{\mathrm{d})}\end{array}$}} & \multirow{2}{*}{\multicolumn{2}{|c|}{$\begin{array}{l}T_{1 / 2}^{(i)} \\
(\mathrm{ps})\end{array}$}} \\
\hline & & & & & & & & & & \\
\hline & & & & $X L$ & Exp..$^{e, f)}$ & Theor. & Exp.g) & Theor. & Exp. ${ }^{h)}$ & Theor. $^{c}$ ) \\
\hline \multirow[t]{2}{*}{50.1} & 29.9 & $B b$ & 20.3 & E1 & $1.16(19) \times 10^{-3}$ & $4.7 \times 10^{-4}$ & $3.2(3)$ & 2.6 & $730(100)$ & 1481 \\
\hline & 0.0 & $B b$ & 50.1 & E1 & $2.4(3) \times 10^{-3}$ & $1.21 \times 10^{-3}$ & $100(2)$ & 100 & & \\
\hline \multirow[t]{3}{*}{61.4} & 29.9 & $b b$ & 31.6 & M1 & $\left.4.1(11) \times 10^{-3} \mathrm{~g}\right)$ & $6.9 \times 10^{-3}$ & $77(10)$ & 135 & $700(180)$ & 630 \\
\hline & & & & E2 & $4.7(12) \times 10^{3 \mathrm{~g})}$ & $4.6 \times 10^{3}$ & & & & \\
\hline & 0.0 & $b b$ & 61.4 & $\mathrm{E} 2$ & $3.0(8) \times 10^{3}$ & $2.8 \times 10^{3}$ & $100(10)$ & 100 & & \\
\hline \multirow[t]{5}{*}{79.7} & 61.4 & $B b$ & 18.3 & E1 & & $1.5 \times 10^{-4}$ & & 0.9 & $240(80)$ & 352 \\
\hline & 50.1 & $B B$ & 29.6 & M1 & & $1.32 \times 10^{-2}$ & & 3.9 & & \\
\hline & & & & E2 & & $1.03 \times 10^{4}$ & & & & \\
\hline & 29.9 & $B b$ & 49.9 & E1 & $1.0(4) \times 10^{-3}$ & $1.1 \times 10^{-4}$ & $26(2)$ & 13.4 & & \\
\hline & 0.0 & $B b$ & 79.7 & E1 & $9.9(33) \times 10^{-4}$ & $2.0 \times 10^{-4}$ & $100(3)$ & 100 & & \\
\hline \multirow[t]{5}{*}{123.8} & 79.7 & $B B$ & 44.1 & M1 & $\left.2.1(6) \times 10^{-3 g}\right)$ & $1.4 \times 10^{-3}$ & $0.5(2)$ & 0.31 & $390(50)$ & 364 \\
\hline & & & & $\mathrm{E} 2$ & $\left.4.1(11) \times 10^{3} \mathrm{~g}\right)$ & $4.0 \times 10^{3}$ & & & & \\
\hline & 61.4 & $B b$ & 62.5 & E1 & $2.4(5) \times 10^{-4}$ & $3.9 \times 10^{-5}$ & $12.6(18)$ & 1.6 & & \\
\hline & 50.1 & $B B$ & 73.6 & E2 & $2.7(6) \times 10^{3}$ & $2.7 \times 10^{3}$ & $1.0(2)$ & 0.75 & & \\
\hline & 29.9 & $B b$ & 93.9 & E1 & $5.5(9) \times 10^{-4}$ & $7.2 \times 10^{-4}$ & $100(4)$ & 100 & & \\
\hline \multirow[t]{4}{*}{130.2} & 123.8 & $b B$ & 6.4 & E1 & & $1.6 \times 10^{-3}$ & & 1.28 & $220(50)$ & 479 \\
\hline & 61.4 & $b b$ & 68.7 & M1 & $\left.1.4(3) \times 10^{-2} \mathrm{~g}\right)$ & $8.8 \times 10^{-3}$ & $76(7)$ & 108 & & \\
\hline & & & & $\mathrm{E} 2$ & $\left.8.7(21) \times 10^{3} \mathrm{~g}\right)$ & $3.1 \times 10^{3}$ & & & & \\
\hline & 29.9 & $b b$ & 100.3 & E2 & $1.02(26) \times 10^{4}$ & $4.2 \times 10^{3}$ & $100(16)$ & 100 & & \\
\hline \multirow[t]{5}{*}{174.58} & 130.2 & $B b$ & 44.4 & E1 & $\leq 8 \times 10^{-5}$ & $2.4 \times 10^{-6}$ & $0.5(5)$ & 0.01 & $200(60)$ & 90 \\
\hline & 123.8 & $B B$ & 50.8 & M1 & $\left.7.4(\overline{32}) \times 10^{-3} \mathrm{~g}\right)$ & $3.3 \times 10^{-2}$ & $1.7(8)$ & 2.1 & & \\
\hline & & & & $\mathrm{E} 2$ & $\left.6.6(29) \times 10^{3} \mathrm{~g}\right)$ & $2.6 \times 10^{3}$ & & & & \\
\hline & 79.7 & $B B$ & 94.9 & $\mathrm{E} 2$ & & $4.0 \times 10^{3}$ & $<2.6$ & 1.0 & & \\
\hline & 61.4 & $B b$ & 113.2 & E1 & $5.2(17) \times 10^{-4}$ & $1.6 \times 10^{-3}$ & $100(5)$ & 100 & & \\
\hline \multirow[t]{4}{*}{174.62} & 174.58 & $b B$ & 0.04 & E1 & & $2.0 \times 10^{-3}$ & & $\sim 0$ & $130(30)$ & 226 \\
\hline & 130.2 & $b b$ & 44.4 & M1 & $\left.2.9(21) \times 10^{-2} \mathrm{~h}\right)$ & $3.2 \times 10^{-2}$ & $8(6)$ & 34 & & \\
\hline & & & & E2 & & $2.3 \times 10^{3}$ & & & & \\
\hline & 61.4 & $b b$ & 113.2 & E2 & $2.4(9) \times 10^{4}$ & $6.4 \times 10^{3}$ & $100(70)$ & 100 & & \\
\hline \multirow[t]{5}{*}{247.4} & 174.62 & $B b$ & 72.8 & E1 & & $1.8 \times 10^{-4}$ & & 4.2 & $150(50)$ & 117 \\
\hline & 174.58 & $B B$ & 72.8 & M1 & & $2.0 \times 10^{-2}$ & & 5.3 & & \\
\hline & & & & $\mathrm{E} 2$ & & $1.8 \times 10^{3}$ & & & & \\
\hline & 130.2 & $B b$ & 117.2 & E1 & $4.8(19) \times 10^{-4}$ & $1.03 \times 10^{-3}$ & $100(5)$ & 100 & & \\
\hline & 123.8 & $B B$ & 123.5 & $(\mathrm{E} 2)$ & $2.6(11) \times 10^{3}$ & $4.7 \times 10^{3}$ & $7.2(14)$ & 6.3 & & \\
\hline \multirow[t]{5}{*}{286.1} & 234.8 & $a b$ & 51.3 & $\mathrm{E} 2$ & & $6.6 \times 10^{-2}$ & & $\sim 0$ & $610(50)$ & 350 \\
\hline & 50.1 & $a B$ & 236.0 & E1 & $2.76(24) \times 10^{-5}$ & $6.0 \times 10^{-5}$ & $100(6)$ & 100 & & \\
\hline & 29.9 & $a b$ & 256.3 & $\mathrm{E} 2$ & $2.41(21) \times 10^{2}$ & $1.6 \times 10^{2}$ & $56.2(9)$ & 17 & & \\
\hline & 0.0 & $a b$ & 286.1 & M1 & $\leq 2.2 \times 10^{-4}$ i) & $4.9 \times 10^{-4}$ & $14.1(7)$ & 16 & & \\
\hline & & & & $\mathrm{E} 2$ & $\left.<3.8 \times 10^{1} \mathrm{j}\right)$ & $2.5 \times 10^{-1}$ & & & & \\
\hline \multirow[t]{9}{*}{329.9} & 286.1 & $A a$ & 43.8 & E1 & $4.5(5) \times 10^{-4}$ & $6.0 \times 10^{-5}$ & $8.0(1)$ & 2.8 & $360(40)$ & 803 \\
\hline & 234.8 & $A c$ & 95.1 & E1 & & $1.1 \times 10^{-7}$ & $<0.5$ & 0.05 & & \\
\hline & 123.8 & $A B$ & 206.1 & E2 & $1.51(17) \times 10^{2}$ & $2.1 \times 10^{1}$ & $8.5(9)$ & 3.3 & & \\
\hline & 79.7 & $A B$ & 250.4 & M1 & $\left.6.5(11) \times 10^{-5} \mathrm{~g}\right)$ & $1.4 \times 10^{-4}$ & $12.8(18)$ & 19 & & \\
\hline & & & & E2 & $\left.7.0(10) \times 10^{1} \mathrm{~g}\right)$ & $1.4 \times 10^{1}$ & & & & \\
\hline & 50.1 & $A B$ & 279.7 & M1 & $\leq 3.7 \times 10^{-5 i)}$ & $9.1 \times 10^{-5}$ & $1.6(2)$ & 25 & & \\
\hline & & & & $\mathrm{E} 2$ & $\left.\leq 7.1^{\mathrm{j}}\right)$ & $1.8 \times 10^{1}$ & & & & \\
\hline & 29.9 & $A b$ & 300.0 & E1 & $1.43 \overline{(16) \times 10^{-5}}$ & $7.0 \times 10^{-6}$ & $81.3(18)$ & 103 & & \\
\hline & 0.0 & $A b$ & 329.9 & E1 & $1.32(15) \times 10^{-5}$ & $5.1 \times 10^{-6}$ & $100(2)$ & 100 & & \\
\hline
\end{tabular}


Table 5 cont.

\begin{tabular}{|c|c|c|c|c|c|c|c|c|c|c|}
\hline \multirow[t]{15}{*}{334.4} & 329.9 & $a A$ & 4.5 & E1 & \multirow{4}{*}{$9.2(10) \times 10^{3}$} & \multicolumn{2}{|l|}{$1.6 \times 10^{-5}$} & \multirow{2}{*}{$\begin{array}{c}\sim 0 \\
0.09\end{array}$} & \multirow[t]{15}{*}{$240(20)$} & \multirow[t]{15}{*}{216} \\
\hline & 286.1 & $a a$ & 48.3 & E2 & & $2.8 \times 10^{3}$ & $0.88(9)$ & & & \\
\hline & 280.2 & $a c$ & 54.2 & M1 & & $2.0 \times 10^{-4}$ & & \multirow[t]{2}{*}{0.06} & & \\
\hline & & & & $\mathrm{E} 2$ & & $7.2 \times 10^{-3}$ & & & & \\
\hline & 234.8 & $a c$ & 99.6 & M1 & & $1.11 \times 10^{-3}$ & & \multirow[t]{2}{*}{2.0} & & \\
\hline & & & & $\mathrm{E} 2$ & & $3.3 \times 10^{-5}$ & & & & \\
\hline & 130.2 & $a b$ & 204.3 & $\mathrm{E} 2$ & $1.37(21) \times 10^{2}$ & $1.7 \times 10^{2}$ & $18(2)$ & 7.6 & & \\
\hline & 123.8 & $a B$ & 210.7 & E1 & $2.25(21) \times 10^{-5}$ & $6.5 \times 10^{-5}$ & $100(2)$ & 100 & & \\
\hline & 79.7 & $a B$ & 254.7 & (E1) & $7.7(8) \times 10^{-6}$ & $1.14 \times 10^{-5}$ & $60(3)$ & 31 & & \\
\hline & 61.4 & $a b$ & 272.9 & M1 & $\left.1.55(16) \times 10^{-5} \mathrm{~g}\right)$ & $6.9 \times 10^{-5}$ & $41(2)$ & \multirow[t]{2}{*}{5.0} & & \\
\hline & & & & E2 & $\left.7.2(7) \times 10^{1} \mathrm{~g}\right)$ & $1.24 \times 10^{1}$ & & & & \\
\hline & 29.9 & $a b$ & 304.5 & M1 & $\left.5.7(6) \times 10^{-4} \mathrm{~g}\right)$ & $7.3 \times 10^{-4}$ & $90(4)$ & \multirow[t]{2}{*}{55} & & \\
\hline & & & & E2 & $\left.6.0(6)^{g}\right)$ & $5.3 \times 10^{1}$ & & & & \\
\hline & 0.0 & $a b$ & 334.4 & M1 & $\left.3.3(3) \times 10^{-4} \mathrm{~g}\right)$ & $1.11 \times 10^{-4}$ & $88(2)$ & \multirow[t]{2}{*}{22} & & \\
\hline & & & & E2 & $\left.1.45(14) \times 10^{1} \mathrm{~g}\right)$ & $2.7 \times 10^{1}$ & & & & \\
\hline \multirow[t]{17}{*}{369.4} & 350.5 & $C A$ & 18.9 & E2 & & $1.34 \times 10^{1}$ & & $\sim 0$ & \multirow[t]{17}{*}{$200(50)$} & \multirow[t]{17}{*}{161} \\
\hline & 342.6 & $C a$ & 26.8 & E1 & & $5.0 \times 10^{-7}$ & & $\sim 0$ & & \\
\hline & 334.4 & $\mathrm{Ca}$ & 35.0 & E1 & & $4.0 \times 10^{-7}$ & & $\sim 0$ & & \\
\hline & 329.9 & $C A$ & 39.5 & M1 & & $5.3 \times 10^{-3}$ & & \multirow[t]{2}{*}{0.74} & & \\
\hline & & & & E2 & & 1.92 & & & & \\
\hline & 280.2 & $C c$ & 89.1 & E1 & $9.1(24) \times 10^{-5}$ & $2.2 \times 10^{-4}$ & $11.9(8)$ & 32 & & \\
\hline & 234.8 & $C c$ & 134.5 & E1 & $2.1(6) \times 10^{-4}$ & $1.9 \times 10^{-4}$ & $96(15)$ & 95 & & \\
\hline & 174.58 & $C B$ & 194.9 & E2 & & 2.3 & & 0.1 & & \\
\hline & 123.8 & $C B$ & 245.5 & M1 & & $9.2 \times 10^{-4}$ & $5(2)$ & \multirow[t]{2}{*}{33} & & \\
\hline & & & & E2 & & $1.19 \times 10^{1}$ & & & & \\
\hline & 79.7 & $C B$ & 289.5 & M1 & & $5.3 \times 10^{-4}$ & & \multirow[t]{2}{*}{34} & & \\
\hline & & & & $\mathrm{E} 2$ & & $1.6 \times 10^{1}$ & & & & \\
\hline & 61.4 & $\mathrm{Cb}$ & 307.9 & E1 & $8.6(24) \times 10^{-6}$ & $2.7 \times 10^{-6}$ & $47(7)$ & 16 & & \\
\hline & 50.1 & $C B$ & 319.3 & M1 & $\left.1.5(4) \times 10^{-3} \mathrm{~g}\right)$ & $1.29 \times 10^{-3}$ & $100(3)$ & \multirow[t]{2}{*}{100} & & \\
\hline & & & & E2 & $6.7(17) \mathrm{g})$ & 9.2 & & & & \\
\hline & 29.9 & $C b$ & 339.5 & E1 & $1.8(5) \times 10^{-6}$ & $1.3 \times 10^{-6}$ & $13.1(12)$ & \multirow{2}{*}{$\begin{array}{c}10.4 \\
39\end{array}$} & & \\
\hline & 0.0 & $\mathrm{Cb}$ & 369.4 & (E1) & $2.0(5) \times 10^{-6}$ & $3.8 \times 10^{-6}$ & $18.8(12)$ & & & \\
\hline
\end{tabular}

a) Band assignment for the initial and final states: $a, b, c, A, B$ and $C$ stand for the $K^{\pi}=1 / 2^{+}, 3 / 2^{+}, 5 / 2^{+}$, $1 / 2^{-}, 3 / 2^{-}$and $5 / 2^{-}$PD bands, respectively.

b) In units of $\mathrm{e}^{2} \cdot \mathrm{fm}^{2}$ for $E 1, \mathrm{e}^{2} \cdot \mathrm{fm}^{4}$ for $E 2$, and $\mu_{N}^{2}$ for $M 1$ transitions; see the $X L$ multipolarity in column 5 .

c) Total conversion coefficients were taken from Refs. [19, 28].

d) $\gamma$-intensity includes the $M 1$ and $E 2$ components for the mixed $M 1 / E 2$ transitions.

e) $\gamma$-branchings are an average of data from [29] and the $\alpha$-decay data in Ref. [19].

f) $T_{1 / 2}$ values are the average of half-lives listed in Refs. [19, 27].

g) $M 1$ and $E 2$ components obtained using the measured $\delta(E 2 / M 1)$ from Ref. [19].

h) $\delta(E 2 / M 1)$ unknown, assuming $|\delta(E 2 / M 1)| \sim 0$.

i) $\delta(E 2 / M 1)$ unknown, assuming $|\delta(E 2 / M 1)| \geq 0$.

j) $\delta(E 2 / M 1)$ unknown, assuming $|\delta(E 2 / M 1)| \leq \infty$. 
Table 6

$\left|D_{0}\right|$ values for the parity-doublet bands in ${ }^{223,227}$ Ra calculated from Eq. (1).

\begin{tabular}{|c|c|c|c|c|c|}
\hline \multirow[t]{3}{*}{$\mathrm{K}^{ \pm}$} & \multirow[t]{3}{*}{$\mathrm{I}_{i}^{\pi} \rightarrow \mathrm{I}_{f}^{\pi}$} & \multicolumn{4}{|c|}{$\left|D_{0}\right|(e \cdot f m)$} \\
\hline & & \multicolumn{2}{|c|}{${ }^{223} \mathrm{Ra}^{\text {a) }}$} & \multicolumn{2}{|c|}{${ }^{227} \mathrm{Ra}^{\mathrm{b}}$} \\
\hline & & Exp. & Theor. ${ }^{c)}$ & Exp. & Theor. ${ }^{c)}$ \\
\hline \multirow[t]{7}{*}{$1 / 2^{ \pm}$} & $1 / 2^{-} \rightarrow 1 / 2^{+}$ & & & $\geq 0.062$ & 0.20 \\
\hline & $1 / 2^{-} \rightarrow 3 / 2^{+}$ & & & $\geq 0.059$ & 0.19 \\
\hline & $3 / 2^{-} \rightarrow 1 / 2^{+}$ & $0.076(4)$ & 0.027 & $\geq 0.12$ & 0.20 \\
\hline & $3 / 2^{-} \rightarrow 3 / 2^{+}$ & & 0.038 & $\geq 0.12$ & 0.15 \\
\hline & $3 / 2^{-} \rightarrow 5 / 2^{+}$ & & 0.013 & $\geq 0.10$ & 0.19 \\
\hline & Representative $^{\mathrm{d})}=$ & 0.08 & & $\geq 0.11$ & \\
\hline & Schematic Model= & 0.04 & & -0.07 & \\
\hline \multirow[t]{16}{*}{$3 / 2^{ \pm}$} & $3 / 2^{-} \rightarrow 3 / 2^{+}$ & $0.129(9)$ & 0.092 & $0.099(3)$ & 0.12 \\
\hline & $3 / 2^{-} \rightarrow 5 / 2^{+}$ & $0.111(9)$ & 0.070 & $0.123(7)$ & 0.11 \\
\hline & $5 / 2^{-} \rightarrow 3 / 2^{+}$ & $0.124(21)$ & 0.056 & $0.096(8)$ & 0.13 \\
\hline & $5 / 2^{-} \rightarrow 5 / 2^{+}$ & $0.130(22)$ & 0.042 & $0.031(3)$ & 0.03 \\
\hline & $5 / 2^{-} \rightarrow 7 / 2^{+}$ & & 0.036 & $0.135(17)$ & 0.08 \\
\hline & $7 / 2^{-} \rightarrow 5 / 2^{+}$ & $0.080(7)$ & 0.092 & & \\
\hline & $7 / 2^{-} \rightarrow 7 / 2^{+}$ & $0.083(9)$ & 0.034 & & \\
\hline & $7 / 2^{-} \rightarrow 9 / 2^{+}$ & & 0.128 & & \\
\hline & $9 / 2^{-} \rightarrow 7 / 2^{+}$ & $0.074(12)$ & 0.130 & & \\
\hline & $9 / 2^{-} \rightarrow 9 / 2^{+}$ & $\leq 0.065$ & 0.011 & & \\
\hline & $9 / 2^{-} \rightarrow 11 / 2^{+}$ & & 0.141 & & \\
\hline & $11 / 2^{-} \rightarrow 9 / 2^{+}$ & $0.069(13)$ & 0.101 & & \\
\hline & $13 / 2^{-} \rightarrow 11 / 2^{+}$ & & 0.126 & & \\
\hline & $13 / 2^{-} \rightarrow 13 / 2^{+}$ & & 0.115 & & \\
\hline & Representative $^{\mathrm{d})}=$ & 0.12 & & 0.10 & \\
\hline & Schematic Model= & 0.03 & & Not provided & \\
\hline \multirow[t]{4}{*}{$5 / 2^{ \pm}$} & $5 / 2^{-} \rightarrow 5 / 2^{+}$ & $0.035(5)$ & 0.033 & & \\
\hline & $5 / 2^{-} \rightarrow 7 / 2^{+}$ & $0.037(5)$ & 0.057 & & \\
\hline & Representative $^{\mathrm{d})}=$ & 0.04 & & & \\
\hline & Schematic Model= & 0.04 & & Not provided & \\
\hline
\end{tabular}

a) Using $B(E 1)$ from Table 5 .

b) Using $B(E 1)$ from Table 4 .

c) Present work. Here $\left|D_{0}\right|$ is not directly calculated, but extracted from Eq. (1)

as a parameter.

d) The representative values are the average of the four largest experimental values, as discussed in the text.

The new experimental data on ${ }^{227}$ Ra show fast intra-PD band $E 1$ transitions, which provide an important confirmation of the PD character for the $K^{\pi}=1 / 2^{ \pm}$and $3 / 2^{ \pm}$bands in ${ }^{227}$ Ra. Previously, the PD character of these bands was recognized [19] largely on the basis of the regional systematics and from the model-dependent arguments.

A high degree of the measured $E 1$ collectivity represents a second important finding. For the $K^{\pi}=1 / 2^{ \pm}$band in ${ }^{227} \mathrm{Ra}$ (see Table 6 ) we obtain an average value of $D_{0} \geq 0.11$ e.fm. This value is based on the $3 / 2^{-}$state for which the lifetime limit is more restrictive than for the $1 / 2^{-}$level. (If we take $T_{1 / 2} \sim 16 \mathrm{ps}$ as the half-life for the $3 / 2^{-}$state, we obtain $D_{0} \sim 0.16 \mathrm{e} \cdot \mathrm{fm}$ for the $K^{\pi}=1 / 2^{ \pm}$band.) In any case, the $D_{0}$ value for the $K^{\pi}=1 / 2^{ \pm}$band is found to be significantly higher in ${ }^{227} \mathrm{Ra}$ than in ${ }^{223} \mathrm{Ra}$.

The $D_{0}$ values for the $K^{\pi}=3 / 2^{ \pm}$bands in ${ }^{223} \mathrm{Ra}$ and ${ }^{227} \mathrm{Ra}$ show strong variation, and thus a single $D_{0}$ value is hardly representative of a band. Nevertheless, in order to have some means of intercomparison, we have arbitrarily selected the weighted average of the four largest values for the lowest members of the band to be the representative value. 
In ${ }^{227} \mathrm{Ra}$ this procedure gives $\left|D_{0}\right| \sim 0.10 \mathrm{e} \cdot \mathrm{fm}$, marginally lower than the equivalent value in ${ }^{223} \mathrm{Ra}(\sim 0.12 \mathrm{e} \cdot \mathrm{fm})$. Note, however, that the average $D_{0}$ value for the higher-lying $7 / 2^{-}, 9 / 2^{-}$, and $11 / 2^{-}$states in ${ }^{223} \mathrm{Ra}$ is only $\sim 0.08 \mathrm{e} \cdot \mathrm{fm}$.

It can be concluded that, on average, the $D_{0}$ values for the $\left(K^{\pi}=1 / 2^{ \pm}\right.$and $\left.3 / 2^{ \pm}\right)$ PD bands in ${ }^{227} \mathrm{Ra}$ and ${ }^{223} \mathrm{Ra}$ are found to be quite similar. They oscillate at $D_{0} \sim$ $0.10 \mathrm{e} \cdot \mathrm{fm}$ - a value quite moderate in comparison with those measured for a narrow sequence of the neighbouring $\mathrm{Ra}$ nuclei, from ${ }^{217} \mathrm{Ra}$ to ${ }^{226} \mathrm{Ra}$, where the values of $D_{0}$ range from as low as $\sim 0.03 \mathrm{e} \cdot \mathrm{fm}$ up to $\sim 0.35 \mathrm{e} \cdot \mathrm{fm}[2]$.

\subsection{Configuration dependence of $D_{0}$}

The results listed in Table 6 verify the existence of a configuration dependence of $D_{0}$ in both ${ }^{223} \mathrm{Ra}$ and ${ }^{227} \mathrm{Ra}$. Further study could establish precisely the magnitude of this effect. Of particular importance are precise lifetime measurements for the $K^{\pi}=1 / 2^{-}$ band in ${ }^{227} \mathrm{Ra}$ and verification of the $D_{0}$ fluctuations observed for the $K^{\pi}=3 / 2^{ \pm}$band in ${ }^{223} \mathrm{Ra}$.

The schematic model calculations [2] predict a pronounced configuration dependence of $D_{0}$ for some odd-A nuclei, such as ${ }^{227} \mathrm{Th}$, but not for ${ }^{223} \mathrm{Ra}$ nor ${ }^{227} \mathrm{Ra}$. However, the purpose of these calculations [2] was to provide a general picture of a region rather than a detailed insight into a given nucleus. Indeed, the model predictions for ${ }^{223,225,227} \mathrm{Ra}$ are well off the mark, being below the measured values by a factor of $2-3$ on average (the calculated results are listed as 'Schematic Model' in Table 6). In particular, the predicted values for the $K^{\pi}=1 / 2^{ \pm}\left({ }^{223} \mathrm{Ra}\right), K^{\pi}=3 / 2^{ \pm}\left({ }^{223} \mathrm{Ra}\right), K^{\pi}=5 / 2^{ \pm}\left({ }^{225} \mathrm{Ra}\right)$ - the result for ${ }^{225} \mathrm{Ra}$ listed here is not shown in Table 6 - and $K^{\pi}=1 / 2^{ \pm}\left({ }^{227} \mathrm{Ra}\right)$ bands are [2] $D_{0}$ $=0.04,0.03,-0.04$, and $-0.07 \mathrm{e} \cdot \mathrm{fm}$, which must be compared to the measured values of $\left|D_{0}\right|=0.08,0.12,0.16$, and $\sim 0.11-0.16 \mathrm{e} \cdot \mathrm{fm}$, respectively. Agreement is found only for the $K^{\pi}=5 / 2$ band in ${ }^{223}$ Ra. Quite clearly, more sophisticated calculations are needed to study this effect.

\subsection{Quenched $E 1$ transitions; $E 1$ transitions outside parity-doublet bands}

In ${ }^{223} \mathrm{Ra}$ (see Table 5), there are two mutually exclusive classes of $E 1$ transitions [17]: a) the fast transitions in the range from $3 \times 10^{-3}$ to $9 \times 10^{-5} \mathrm{e}^{2} \cdot \mathrm{fm}^{2}$, and b) the slow transitions in the range from $3 \times 10^{-5}$ to $2 \times 10^{-6} \mathrm{e}^{2} \cdot \mathrm{fm}^{2}$. It is remarkable that all the $E 1$ transitions between members of the same PD band (intra-PD transitions) belong exclusively to class ' $a$ ', while all the transitions between different PD bands (inter-PD) belong exclusively to class ' $b$ '. Although most of the $E 1$ transitions in ${ }^{227}$ Ra seem to follow this double classification pattern, the situation seems to be somewhat different from that in ${ }^{223}$ Ra. Our study has firmly established a few exceptions to this pattern in ${ }^{227}$ Ra. These exceptions are discussed next.

The first case involves the 'exceptionally' fast $131.0-\mathrm{keV}$ transition from the $3 / 2^{-}$ state at $284.3 \mathrm{keV}$ (a member of the $K^{\pi}=1 / 2^{ \pm}$band) to a level outside the PD structure (see Table 4). Note that the parity-changing 131.0-keV transition de-excites the $3 / 2^{-}$state and feeds the $153.3-\mathrm{keV}$ state with the proposed spin and parity of $3 / 2^{+}, 5 / 2^{+}$, or $7 / 2^{+}$. The level-lifetime limit implies this transition to be $E 1$ (with a weak $M 2$ component if any), thus excluding the spin $7 / 2^{+}$for the $153.3-\mathrm{keV}$ state.

The second case involves two $E 1$ transitions (of energy 76.1 and $100.1 \mathrm{keV}$, respectively) de-exciting the same level: the $5 / 2^{-}$state at $102 \mathrm{keV}$ (see Table 4 ). The first transition, classified as an intra-PD, is quenched with the $B(E 1)$ rate almost two orders of magnitude lower than for the other transitions in this PD. The second one, classified as 
an inter-PD transition, is unexpectedly enhanced by almost the same order of magnitude. Since both transitions feed states with spin and parity $5 / 2^{+}$, this double effect seems to point towards some mixing of the states involved. However, this mixing does not seem to affect the equivalent $E 1$ transitions (of energy 64.3 and $88.3 \mathrm{keV}$ ) from the lower state of the same $\left(K^{\pi}=3 / 2^{-}\right)$PD band which populate these two $5 / 2^{+}$states with the fast and slow rates as 'expected' for the intra- and inter-PD transitions, respectively. As part of the explanation, note that the $K^{\pi}=3 / 2^{-}$PD band classified as $3 / 2[761]$ was found $[19,23]$ to be strongly mixed with other bands by the Coriolis interaction. In order to discuss the aforementioned issues in the framework of our theoretical calculations, a brief description of the model is given below.

\section{$5 \quad$ Description of the model}

\subsection{Outline of the Hamiltonian}

For the theoretical description of the low-lying states in ${ }^{227} \mathrm{Ra}$ and ${ }^{223} \mathrm{Ra}$ we take the standard axially symmetric rotor model [30] including Coriolis coupling, $H_{\text {rot }}$, with the intrinsic degrees of freedom of the nucleus, $H_{\text {intr }}$, described by the quasiparticle-phonon model (QPM) [31]. Thus, the total Hamiltonian $H$ is

$$
H=H_{\text {intr }}+H_{\text {rot }},
$$

where $H_{\text {rot }}$ is given by

$$
H_{\text {rot }}=\frac{\hbar^{2}}{2 \Im}\left[\hat{I}^{2}+\hat{\jmath}^{2}-{\hat{I_{3}}}^{2}-{\hat{\jmath_{3}}}^{2}\right]-\frac{\hbar^{2}}{2 \Im}\left(\hat{J_{+}} \hat{\jmath_{-}}+\hat{J_{-}} \hat{\jmath_{+}}\right) .
$$

Here, $\Im$ is the moment of inertia with respect to the axis perpendicular to the symmetry axis; $\hat{I}_{3}$ and $\hat{\jmath}_{3}$ are the projection operators of the total and intrinsic angular momenta $\vec{I}$ and $\vec{\jmath}$ onto the symmetry axis; $\hat{I}_{ \pm}=\hat{I}_{1} \pm i \hat{I}_{2}$ and $\hat{\jmath_{ \pm}}=\hat{\jmath}_{1} \pm i \hat{\jmath}_{2}$. The inclusion of the Coriolis coupling term requires the matrix of the total Hamiltonian (2) to be constructed and diagonalized within the space of symmetrized functions [32]:

$$
|I M K \rho\rangle=\left[\frac{2 I+1}{16 \pi}\right]^{1 / 2}\left\{D_{M K}^{I}|K, \rho\rangle+(-1)^{I} D_{M-K}^{I} R_{1}(\pi)|K, \rho\rangle\right\} .
$$

In this equation $D_{M K}^{I}$ are the Wigner functions of the Euler angles, $|K, \rho\rangle$ is the eigenvector of the intrinsic Hamiltonian satisfying $H_{\text {intr }}|K, \rho\rangle=E_{\rho}^{(\mathrm{intr})}|K, \rho\rangle$, and $\rho$ are the quantum numbers of a given intrinsic state with the projection $K$ of the intrinsic angular momentum onto the symmetry axis. $R_{1}(\pi)$ is a rotation by angle $\pi$ around the first intrinsic axis. After diagonalizing the total Hamiltonian matrix we obtain the energy levels $E_{q}(I)$ and the corresponding wave functions:

$$
|I M q\rangle=\sum_{K \rho} b_{K \rho}^{I q}|I M K \rho\rangle,
$$

where the index $q$ characterizes a given rotational band.

We approximate the intrinsic Hamiltonian by the QPM Hamiltonian [31, 33]:

$$
H_{\mathrm{intr}} \approx H_{\mathrm{QPM}}, \quad|K, \rho\rangle \approx\left|\Psi_{K}(\rho)\right\rangle,
$$

where $\left|\Psi_{K}(\rho)\right\rangle$ are the eigenvectors of the QPM Hamiltonian, $H_{\mathrm{QPM}}\left|\Psi_{K}(\rho)\right\rangle=\eta_{\rho}\left|\Psi_{K}(\rho)\right\rangle$. $H_{\mathrm{QPM}}$ involves the average field, the pairing residual interaction and the long-range residual interactions in quadrupole-quadrupole and octupole-octupole form [31, 33], which also 
include the coupling between octupole and quasiparticle degrees of freedom. The intrinsic wave function $\left|\Psi_{K}(\rho)\right\rangle$ of an odd-A nucleus has one-quasiparticle and one-quasiparticleplus-phonon components [31] given by

$$
\left|\Psi_{K}(\rho)\right\rangle=\left[\sum_{\nu} C_{\nu}^{\rho} \alpha_{\tilde{\nu}}^{\dagger}+\sum_{\tilde{g} \tilde{\nu}} D_{\tilde{\nu} \tilde{g}}^{\rho} \delta_{\tilde{\mu}+\tilde{K}_{\nu}, \tilde{K}_{\rho}} \alpha_{\tilde{\nu}}^{\dagger} Q_{\tilde{g}}^{\dagger}\right]|\rangle,
$$

where $\alpha_{\tilde{\nu}}^{\dagger}=\alpha_{\nu \sigma_{\nu}}^{\dagger}$ represents the quasiparticle-creation operator $\left(\sigma_{\nu}= \pm 1\right.$ is the sign of the projection of the intrinsic angular momentum onto the symmetry axis). The first term in Eq. (7) represents the sum over the one-quasiparticle components of valence quasiparticles which can be occupied by an odd nucleon, and $C_{\nu}^{\rho}$ is the amplitude of each one-quasiparticle component. The second term in Eq. (7) involves the sum over all quasiparticle-plus-phonon components. $Q_{\tilde{g}}^{\dagger}=Q_{\lambda, \mu \sigma, i}^{\dagger}$ stands for the phonon-creation operator of the vibrating nuclear core with the multipolarity $\lambda$ and multipolarity projection $\tilde{\mu}=\mu \sigma(\mu \geq 0, \sigma= \pm 1)$. The index $i$ enumerates the phonons with the same $\lambda \mu$. $D_{\tilde{\nu} \tilde{g}}^{\rho}$ represents the amplitude of the quasiparticle-plus-phonon component $\nu g$ in the wave function (7). In each one-quasiparticle-plus-phonon component, the angular momentum projection $\tilde{\mu}$ of the core phonon couples with the quasiparticle angular momentum projection $\tilde{K}_{\nu}$ to the intrinsic angular momentum projection $\tilde{K}_{\rho}\left(\tilde{K}_{\rho}=\tilde{\mu}+\tilde{K}_{\nu}\right)$. The symbol |\rangle stands for the product of the phonon vacuum and the vacuum for valence quasiparticles which can be occupied by an odd nucleon (we assume that valence quasiparticles do not enter the structure of the core phonons). The structure of the phonon-creation operator $Q_{\tilde{g}}^{\dagger}$ is given by a superposition of two-quasiparticle states [31, 34]:

$$
Q_{\tilde{g}}^{\dagger}=Q_{\lambda, \mu \sigma, i}^{\dagger}=\frac{1}{2} \sum_{q q^{\prime}}\left(\Psi_{q q^{\prime}}^{\lambda \mu i} A^{\dagger}\left(q q^{\prime} ; \mu \sigma\right)-\phi_{q q^{\prime}}^{\lambda \mu i} A^{\dagger}\left(q q^{\prime} ; \mu \sigma\right)\right)
$$

where $A^{\dagger}\left(q q^{\prime} ; \mu \sigma\right)$ are two-quasiparticle-creation operators

$$
A^{\dagger}\left(q q^{\prime} ; \mu \sigma\right)=\sum_{\sigma^{\prime}= \pm 1} \delta_{\sigma^{\prime}\left(K_{q} \pm K_{q^{\prime}}\right), \sigma \mu} \alpha_{q^{\prime} \sigma^{\prime}}^{\dagger} \alpha_{q \sigma^{\prime}}^{\dagger}
$$

In Eq. (8) $\Psi_{q q^{\prime}}^{\lambda \mu i}$ and $\phi_{q q^{\prime}}^{\lambda \mu i}$ represent the amplitudes of given two-quasiparticle state $q q^{\prime}$ in the phonon $Q_{\tilde{g}}^{\dagger}$. Expressions for these amplitudes, for the amplitudes $C_{\nu}^{\rho}$ and $D_{\tilde{\nu} \tilde{g}}^{\rho}$ in the wave function (7), and for the corresponding energy $\eta_{\rho}$ can be found in Refs. [31, 34].

\subsection{Transition probabilities}

The reduced probability $B(X L ; i \rightarrow f)$ is given by the reduced matrix element $\left\langle I_{f} q_{f}\|M(X L)\| I_{i} q_{i}\right\rangle$ of the multipole operator $M(X L \mu)$ of multipolarity $L$ and type $X$, between the final and initial states $\left|I_{f} q_{f}\right\rangle$ and $\left|I_{i} q_{i}\right\rangle$, respectively (see for example [32]):

$$
B(X L ; i \rightarrow f)=\frac{1}{2 I_{i}+1}\left|\left\langle I_{f} q_{f}\|M(X L)\| I_{i} q_{i}\right\rangle\right|^{2} .
$$

If Coriolis coupling is taken into account and initial and final states are described by the wave function (5), the reduced matrix elements $\left\langle I_{f} q_{f}\|M(X L)\| I_{i} q_{i}\right\rangle$ can be written in the form

$$
\left\langle I_{f} q_{f}\|M(X L)\| I_{i} q_{i}\right\rangle=\sum_{K_{i} \rho_{i}, K_{f} \rho_{f}} b_{K_{i} \rho_{i}}^{I_{i} q_{i}} b_{K_{f} \rho_{f}}^{I_{f} q_{f}}\left\langle I_{f} K_{f} \rho_{f}\|M(X L)\| I_{i} K_{i} \rho_{i}\right\rangle
$$


In the case of axial symmetry without an odd-multipolarity deformation in the average field we obtain [31] (using the Condon-Shortley phase convention)

$$
\begin{aligned}
& \left\langle I_{f} K_{f} \rho_{f} \| M(X L)|| I_{i} K_{i} \rho_{i}\right\rangle= \\
& \quad \sqrt{2 I_{i}+1}\left\{\left(I_{i} K_{i} L K_{f}-K_{i} \mid I_{f} K_{f}\right)\left\langle\Psi_{K_{f}}\left(\rho_{f}\right)\left|M\left(X L K_{f}-K_{i}\right)\right| \Psi_{K_{i}}\left(\rho_{i}\right)\right\rangle+\right. \\
& \left.\quad(-1)^{I_{i}+K_{i}} p_{i}\left(I_{i}-K_{i} L K_{i}+K_{f} \mid I_{f} K_{f}\right)\left\langle\Psi_{K_{f}}\left(\rho_{f}\right)\left|M\left(X L K_{i}+K_{f}\right)\right| \overline{\Psi_{K_{i}}\left(\rho_{i}\right)}\right\rangle\right\}
\end{aligned}
$$

where $\left(I_{i} K_{i} L \mu \mid I_{f} K_{f}\right)$ are Clebsch-Gordan coefficients, $\left|\overline{\Psi_{K_{i}}\left(\rho_{i}\right)}\right\rangle$ is the time reversal of $\left|\Psi_{K_{i}}\left(\rho_{i}\right)\right\rangle$, and $p_{i}= \pm 1$ is the parity of the initial state. Since only $E 1, E 2$, and $M 1$ transitions dominate the low-lying states, the corresponding multipole operators are [32]

$$
\begin{gathered}
\hat{M}(E, L \mu)=\sum_{i=1}^{A} e^{(i)}(L) r^{(i)^{L}} Y_{L \mu}\left(\theta_{i}, \phi_{i}\right), e^{(i)}(L)=e e_{\mathrm{eff}}^{(i)}(L) \\
\hat{M}(M, 1 \mu)=\sqrt{\frac{3}{4 \pi} \sum_{i=1}^{A} \mu_{n}\left[g_{l}^{(i)} \hat{l}_{\mu}^{(i)}+g_{s}^{(i)} \hat{s}_{\mu}^{(i)}\right]+\sqrt{\frac{3}{4 \pi}} \mu_{N} g_{R} \hat{R}_{\mu},}
\end{gathered}
$$

where $e_{\mathrm{eff}}^{(i)}(L)$ is the effective charge of the $i$-th nucleon for multipolarity $L, g_{l}^{(i)}$ and $g_{s}^{(i)}$ are the orbital and spin gyromagnetic ratios, $\mu_{N}$ is the nuclear magneton, $g_{R}$ is the gyromagnetic ratio for the rotational contribution $\left(\hat{R}_{\mu}=\hat{I}_{\mu}-\hat{j}_{\mu}\right.$ are the components of the rotational angular momentum), and $\hat{l}$ and $\hat{s}$ are orbital and spin angular-momentum operators.

The intrinsic matrix elements $\left\langle\Psi_{K_{f}}\left(\rho_{f}\right)|M(X, L \mu)| \Psi_{K_{i}}\left(\rho_{i}\right)\right\rangle$ in Eq. (12) can be obtained by substituting the wave functions $(7)$ for the initial and final states. In the case of the $E L$ transitions,

$$
\begin{aligned}
\left\langle\Psi_{K_{f}}\left(\rho_{f}\right)\right| & M(E, L \mu)\left|\Psi_{K_{i}}\left(\rho_{i}\right)\right\rangle= \\
& \delta_{\rho_{i} \rho_{f}} \delta_{K_{i} K_{f}}\langle|M(E, L \mu)|\rangle \\
& +\sum_{q_{i} q_{f}} C_{q_{f}}^{\rho_{f}^{*}} C_{q_{i}}^{\rho_{i}}\left\langle\left|\alpha_{\tilde{q}_{f}} M(E, L \mu) \alpha_{\tilde{q}_{i}}^{\dagger}\right|\right\rangle \\
& +\sum_{\tilde{q}_{i}} \sum_{\tilde{g}_{f}, \tilde{q}_{f}} D_{\tilde{q}_{f} \tilde{g}_{f}}^{\rho_{f}^{*}} C_{q_{i}}^{\rho_{i}} \delta_{\tilde{\mu}_{f}+\tilde{K}_{q_{f}}, \tilde{K}_{\rho_{f}}}\left\langle\left|Q_{\tilde{g}_{f}} \alpha_{\tilde{q}_{f}} M(E, L \mu) \alpha_{\tilde{q}_{i}}^{\dagger}\right|\right\rangle \\
& +\sum_{\tilde{q}_{f}} \sum_{\tilde{g}_{i} \tilde{q}_{i}} C_{q_{f}^{*}}^{\rho_{f}^{*}} D_{\tilde{q}_{i} \tilde{g}_{i}}^{\rho_{i}} \delta_{\tilde{\mu}_{i}+\tilde{K}_{q_{i}}, \tilde{K}_{\rho_{i}}}\left\langle\left|\alpha_{\tilde{q}_{f}} M(E, L \mu) \alpha_{\tilde{q}_{i}}^{\dagger} Q_{\tilde{g}_{i}}^{\dagger}\right|\right\rangle \\
& +\sum_{\tilde{q}_{i} \tilde{g}_{i}} \sum_{\tilde{q}_{f} \tilde{g}_{f}} D_{\tilde{q}_{f} \tilde{g}_{f}}^{\rho_{f}^{*}} D_{q_{i} g_{i}}^{\rho_{i}} \delta_{\tilde{\mu}_{i}+\tilde{K}_{q_{i}}, \tilde{K}_{i}} \delta_{\tilde{\mu}_{f}+\tilde{K}_{q_{f}}, \tilde{K}_{f}}\left\langle\left|Q_{\tilde{g}_{f}} \alpha_{\tilde{q}_{f}} M(E, L \mu) \alpha_{\tilde{q}_{i}}^{\dagger} Q_{\tilde{g}_{i}}^{\dagger}\right|\right\rangle .
\end{aligned}
$$

If for valence quasiparticle-creation operators $\alpha_{\tilde{q}_{f}}^{\dagger}$ or $\alpha_{\tilde{q}_{i}}^{\dagger}$ we suppose $\left[\alpha_{\tilde{q}_{i}}, Q_{\tilde{g}}\right]=$ $\left[\alpha_{\tilde{q}_{f}}, Q_{\tilde{g}}\right]=0$, then 


$$
\begin{aligned}
& \left\langle\left|\alpha_{\tilde{q}_{f}} M(E, L \mu) \alpha_{\tilde{q}_{i}}^{\dagger}\right|\right\rangle=e^{(\tau)}(L) V_{q_{i} q_{f}}^{(-)}\left\langle\tilde{q}_{f}|M(E, L \mu)| \tilde{q}_{i}\right\rangle \\
& \left\langle\left|Q_{\tilde{g}_{f}} \alpha_{\tilde{q}_{f}} M(E, L \mu) \alpha_{\tilde{q}_{i}}^{\dagger} Q_{\tilde{g}_{i}}^{\dagger}\right|\right\rangle \approx e^{(\tau)}(L) V_{q_{i}}^{(-)}\left\langle\tilde{q}_{f}|M(E, L \mu)| \tilde{q}_{i}\right\rangle \delta_{\tilde{g}_{i}, \tilde{g}_{f}} \\
& \left\langle\left|Q_{\tilde{g}_{f}} \alpha_{\tilde{q}_{f}} M(E, L \mu) \alpha_{\tilde{q}_{q^{\prime}}}^{\dagger}\right|\right\rangle \approx \\
& \quad \frac{1}{2} \sum_{\tau_{\text {neutron }}^{\text {proton }}} e^{(\tau)}(L) \sum_{q_{1} q_{2} \epsilon \tau}\left\langle\tilde{q}_{1}|M(E, L \mu)| \tilde{q}_{2}\right\rangle U_{q_{1} q_{2}}^{(+)}\left(\Psi_{q_{1} q_{2}}^{\lambda_{f} \mu_{f} i_{f}}+\phi_{q_{1} q_{2}}^{\lambda_{f} \mu_{f} i_{f}}\right) \delta_{\tilde{q}_{i} \tilde{q}_{f}} \\
& \left\langle\left|\alpha_{\tilde{q}_{f}} M(E, L \mu) \alpha_{\tilde{q}_{i}}^{\dagger} Q_{\tilde{g}_{i}}^{\dagger}\right|\right\rangle \approx \\
& \quad \frac{1}{2} \sum_{\tau_{\text {neutron }}^{\text {proton }}}^{(\tau)}(L) \sum_{q_{1} q_{2} \epsilon \tau}^{(\tau)}\left\langle\tilde{q}_{1}|M(E, L \mu)| \tilde{q}_{2}\right\rangle U_{q_{1} q_{2}}^{(+)}\left(\Psi_{q_{1} q_{2}}^{\lambda_{i} \mu_{i_{i}} i_{i}}+\phi_{q_{1} q_{2}}^{\lambda_{f} \mu_{f} i_{f}}\right) \delta_{\tilde{q}_{i} \tilde{q}_{f}},
\end{aligned}
$$

where

$$
V_{q_{1} q_{2}}^{(-)}=U_{q_{1}} U_{q_{2}}-V_{q_{1}} V_{q_{2}} \quad, \quad U_{q_{1} q_{2}}^{(+)}=U_{q_{1}} V_{q_{2}}+U_{q_{2}} V_{q_{1}},
$$

and $V_{q}, U_{q}$ are the pairing occupation amplitudes. In Eq. (15), $\left\langle\tilde{q}_{1}|M(E, L \mu)| \tilde{q}_{2}\right\rangle$ are the single-particle matrix elements of the operator $\hat{M}(E, L \mu)$. Similar expressions can be obtained for the intrinsic matrix element of the operator $\hat{M}(M, 1 \mu)$. For the intra-band $E 2$ transitions we used the following expression for \langle|$M(E, 20|\rangle$ in Eq. (14):

$$
\langle| M\left(E 2,20|\rangle=\sqrt{\frac{5}{16 \pi}} Q_{0},\right.
$$

where $Q_{0}$ is the intrinsic quadrupole moment of ${ }^{227} \mathrm{Ra}$ (or ${ }^{223} \mathrm{Ra}$ ).

\subsection{Parameters of the calculations}

The theoretical calculations involved the following four steps:

1. The eigenvalue problem $H_{\mathrm{QPM}}\left|\Psi_{K}(\rho)\right\rangle=\eta_{\rho}\left|\Psi_{K}(\rho)\right\rangle$ was solved, and the QPM intrinsic energies $\eta_{\rho}$ and corresponding amplitudes $C_{\nu}^{\rho}$ and $D_{\nu g}^{\rho}$ were obtained. The Nilsson single-particle average field was used with its standard parametrization [35]. Deformation parameters were $\beta_{2}=0.15, \beta_{4}=0.08$ for ${ }^{227} \mathrm{Ra}$ and $\beta_{2}=0.15, \beta_{4}=0.06$ for ${ }^{223} \mathrm{Ra}$ [35]. All Nilsson shells with $N=2-8$ were included in the QPM calculations. The pairing gap parameters taken from Ref. [31] were $\Delta_{\mathrm{p}}=0.8 \mathrm{MeV}$ for protons and $\Delta_{\mathrm{n}}=1.0 \mathrm{MeV}$ for neutrons. Fermi energies for proton and neutron systems were chosen in order to give the correct number of particles in each of these systems and were $\lambda_{\mathrm{p}}=42.760 \mathrm{MeV}, \lambda_{\mathrm{n}}=$ $49.750 \mathrm{MeV}$ for ${ }^{227} \mathrm{Ra}$ and $\lambda_{\mathrm{p}}=43.220 \mathrm{MeV}, \lambda_{\mathrm{n}}=48.985 \mathrm{MeV}$ for ${ }^{223} \mathrm{Ra}$. All parameters involved in the long-range residual interactions of $H_{\mathrm{QPM}}$ were determined by the energies of quadrupole and octupole core vibrational energies, which in the case of ${ }^{227} \mathrm{Ra}$ were taken from ${ }^{226} \mathrm{Ra}[36,37]: E_{0+}=650 \mathrm{keV}, E_{2+}=846 \mathrm{keV}\left(E_{2+}\right.$ is not available in ${ }^{226} \mathrm{Ra}$, thus it was taken from ${ }^{228} \mathrm{Ra}$ ), $E_{0-}=254 \mathrm{keV}$, and $E_{1_{-}}=1049 \mathrm{keV}$. In the case of ${ }^{223} \mathrm{Ra}$ they were taken as an average of the available data for ${ }^{222} \mathrm{Ra}$ and ${ }^{224} \mathrm{Ra}$ [37]: $E_{0+}=$ $914 \mathrm{keV}, E_{2+}=966 \mathrm{keV}, E_{0-}=234 \mathrm{keV}$, and $E_{1-}=1054 \mathrm{keV}$. The results of the QPM calculations are presented in Tables 7 and 8 for ${ }^{227} \mathrm{Ra}$ and ${ }^{223} \mathrm{Ra}$, respectively. The first two columns contain the description and percentage of the dominant one-quasiparticle $\left(\left|C_{\nu}^{\rho}\right|^{2}\right)$ and quasiparticle-plus-phonon $\left(\left|D_{g}^{\rho}\right|^{2}\right)$ components. The third column presents the experimental bandhead energy of the rotational band built on the corresponding intrinsic state. The fourth column shows the calculated intrinsic energies $\eta_{\rho}$ relative to the intrinsic ground-state energy $\eta_{\rho_{0}}$. 
Table 7

Structure of the low-lying intrinsic states in ${ }^{227} \mathrm{Ra}$.

\begin{tabular}{|c|c|c|c|c|}
\hline \multicolumn{2}{|c|}{$\begin{array}{l}\text { Structure of the } \\
\text { intrinsic state } \\
\left|\Psi_{K}(\rho)\right\rangle \quad \%\end{array}$} & $\begin{array}{c}\text { Bandhead energy } \\
\text { (experimental) } \\
(\mathrm{keV})\end{array}$ & $\begin{array}{c}\text { Intrinsic energy } \\
(\text { theoretical) } \\
\eta-\eta_{0}(\mathrm{keV})\end{array}$ & $\begin{array}{c}E_{\rho}^{(\text {intr })}-E_{\rho_{0}}^{\text {(intr) }} \\
(\text { theoretical }) \\
(\mathrm{keV})\end{array}$ \\
\hline $3 / 2[642]$ & 36.9 & & & \\
\hline $3 / 2[631]$ & 34.4 & & & \\
\hline $3 / 2[761]+Q_{30}^{+}$ & 14.7 & & & \\
\hline $3 / 2[512]+Q_{30}^{+}$ & 3.9 & & & \\
\hline $3 / 2[532]+Q_{30}^{+}$ & 1.1 & 0.0 & 0.0 & 0.0 \\
\hline $3 / 2[761]$ & 80.2 & & & \\
\hline $3 / 2[642]+Q_{30}^{+}$ & 14.1 & & & \\
\hline $3 / 2[651]+Q_{30}^{+}$ & 1.9 & & & \\
\hline $7 / 2[743]-Q_{22}^{+}$ & 0.8 & 90.0 & 84.5 & -15.0 \\
\hline $5 / 2[633]$ & 72.1 & & & \\
\hline $5 / 2[752]+Q_{30}^{+}$ & 14.9 & & & \\
\hline $1 / 2[631]+Q_{22}^{+}$ & 1.8 & & & \\
\hline $11 / 2[725]-Q_{33}^{+}$ & 1.1 & & & \\
\hline $5 / 2[503]+Q_{30}^{+}$ & 1.1 & 1.7 & 32.7 & -112.0 \\
\hline $5 / 2[752]$ & 65.9 & & & \\
\hline $5 / 2[503]$ & 1.8 & & & \\
\hline $5 / 2[633]+Q_{30}^{+}$ & 27.5 & & & \\
\hline $5 / 2[743]+Q_{20}^{+}$ & 0.9 & & 304.0 & \\
\hline $1 / 2[651]$ & 52.9 & & & \\
\hline $1 / 2[631]$ & 10.7 & & & \\
\hline $1 / 2[640]$ & 4.0 & & & \\
\hline $1 / 2[770]+Q_{30}^{+}$ & 11.8 & & & \\
\hline $1 / 2[541]+Q_{30}^{+}$ & 3.6 & & & \\
\hline $1 / 2[521]+Q_{30}^{+}$ & 3.1 & & & \\
\hline $1 / 2[631]+Q_{20}^{+}$ & 1.9 & 120.7 & 121.0 & 184.7 \\
\hline $1 / 2[770]$ & 75.0 & & & \\
\hline $1 / 2[501]$ & 1.0 & & & \\
\hline $1 / 2[651]+Q_{30}^{+}$ & 10.1 & & & \\
\hline $1 / 2[640]+Q_{30}^{+}$ & 5.7 & & & \\
\hline $1 / 2[660]+Q_{30}^{+}$ & 4.3 & & & \\
\hline $1 / 2[631]+Q_{30}^{+}$ & 0.6 & $284.3\left(3 / 2^{-}\right)$ & 176.3 & 274.1 \\
\hline $3 / 2[631]$ & 53.9 & & & \\
\hline $3 / 2[642]$ & 20.7 & & & \\
\hline $3 / 2[761]+Q_{30}^{+}$ & 16.1 & & & \\
\hline $3 / 2[501]+Q_{30}^{+}$ & 1.4 & & & \\
\hline $3 / 2[642]+Q_{20}^{+}$ & 1.0 & & & \\
\hline $3 / 2[631]+Q_{20}^{+}$ & 0.9 & & $\sim 300$ & \\
\hline $3 / 2[501]$ & 56.6 & & & \\
\hline $3 / 2[631]+Q_{30}^{+}$ & 29.2 & & & \\
\hline $1 / 2[501]-Q_{22}^{+}$ & 11.1 & & & \\
\hline $3 / 2[512]+Q_{20}^{+}$ & 1.0 & & $\sim 530$ & \\
\hline $1 / 2[640]$ & 67.4 & & & \\
\hline $1 / 2[651]$ & 1.3 & & & \\
\hline $1 / 2[631]$ & 1.1 & & & \\
\hline $1 / 2[770]+Q_{30}^{+}$ & 16.5 & & & \\
\hline $1 / 2[510]+Q_{30}^{+}$ & 4.8 & & & \\
\hline $1 / 2[530]+Q_{30}^{+}$ & 2.6 & & & \\
\hline $3 / 2[642]-Q_{22}^{+}$ & 0.8 & $731.7\left(3 / 2^{+}\right)$ & 277.0 & 740.0 \\
\hline
\end{tabular}


Table 7 cont.

\begin{tabular}{|lcccc|}
\hline $1 / 2[501]$ & 75.9 & & & \\
$1 / 2[770]$ & 1.0 & & & \\
$5 / 2[503]-Q_{22}^{+}$ & 10.5 & & & \\
$3 / 2[501]-Q_{22}^{+}$ & 7.2 & & & \\
$1 / 2[640]+Q_{30}^{+}$ & 1.2 & & & \\
$1 / 2[631]+Q_{30}^{+}$ & 1.0 & 675.9 & 277.8 & 369.2 \\
\hline $7 / 2[624]$ & 66.7 & & & \\
$7 / 2[743]+Q_{30}^{+}$ & 21.8 & & & \\
$13 / 2[716]-Q_{33}^{+}$ & 3.5 & & & \\
$11 / 2[725]-Q_{32}^{+}$ & 1.3 & & & \\
$3 / 2[621]+Q_{22}^{+}$ & 1.3 & & & \\
$7 / 2[734]+Q_{30}^{+}$ & 1.2 & & & \\
$7 / 2[743]$ & 62.9 & & & \\
$7 / 2[624]+Q_{30}^{+}$ & 27.8 & & \\
$3 / 2[761]+Q_{22}^{+}$ & 3.5 & & \\
$5 / 2[633]+Q_{31}^{+}$ & 2.2 & & \\
\end{tabular}


Table 8

Structure of the low-lying intrinsic states in ${ }^{223} \mathrm{Ra}$.

\begin{tabular}{|c|c|c|c|c|}
\hline \multicolumn{2}{|c|}{$\begin{array}{l}\text { Structure of the } \\
\text { intrinsic state }\end{array}$} & $\begin{array}{c}\text { Bandhead energy } \\
\text { (experimental) } \\
(\mathrm{keV})\end{array}$ & $\begin{array}{c}\text { Intrinsic energy } \\
\text { (theoretical) } \\
\eta-\eta_{0}(\mathrm{keV})\end{array}$ & $\begin{array}{c}E_{\rho}^{(\text {intr })}-E_{\rho_{0}}^{\text {(intr) }} \\
\quad(\text { theoretical }) \\
(\mathrm{keV})\end{array}$ \\
\hline $3 / 2[642]$ & 69.2 & & & \\
\hline $3 / 2[631]$ & 0.8 & & & \\
\hline $3 / 2[761]+Q_{30}^{+}$ & 20.8 & & & \\
\hline $3 / 2[512]+Q_{30}^{+}$ & 3.2 & & & \\
\hline $3 / 2[751]+Q_{30}^{+}$ & 1.1 & 0.0 & 0.0 & 0.0 \\
\hline $3 / 2[761]$ & 62.3 & & & \\
\hline $3 / 2[642]+Q_{30}^{+}$ & 33.7 & & & \\
\hline $1 / 2[770]-Q_{22}^{+}$ & 1.5 & & & \\
\hline $7 / 2[743]-Q_{22}^{+}$ & 0.5 & 50.1 & 290.2 & 264.2 \\
\hline $5 / 2[633]$ & 67.1 & & & \\
\hline $5 / 2[752]+Q_{30}^{+}$ & 25.1 & & & \\
\hline $1 / 2[631]+Q_{22}^{+}$ & 1.0 & & & \\
\hline $11 / 2[725]-Q_{33}^{+}$ & 0.9 & & & \\
\hline $9 / 2[734]-Q_{32}^{+}$ & 0.9 & 234.8 & 401.6 & 357.1 \\
\hline $5 / 2[752]$ & 47.4 & & & \\
\hline $5 / 2[503]$ & 2.5 & & & \\
\hline $5 / 2[633]+Q_{30}^{+}$ & 44.6 & & & \\
\hline $1 / 2[770]+Q_{22}^{+}$ & 1.9 & & & \\
\hline $3 / 2[642]+Q_{31}^{+}$ & 1.3 & 369.4 & 661.8 & 706.6 \\
\hline $1 / 2[640]$ & 48.0 & & & \\
\hline $1 / 2[651]$ & 33.9 & & & \\
\hline $1 / 2[770]+Q_{30}^{+}$ & 5.1 & & & \\
\hline $1 / 2[510]+Q_{30}^{+}$ & 3.1 & & & \\
\hline $1 / 2[750]+Q_{30}^{+}$ & 2.3 & & & \\
\hline $1 / 2[521]+Q_{30}^{+}$ & 2.2 & 286.1 & 285.3 & 292.1 \\
\hline $1 / 2[770]$ & 66.4 & & & \\
\hline $1 / 2[501]$ & 6.3 & & & \\
\hline $1 / 2[640]+Q_{30}^{+}$ & 16.7 & & & \\
\hline $3 / 2[761]-Q_{22}^{+}$ & 2.9 & & & \\
\hline $1 / 2[651]+Q_{30}^{+}$ & 1.4 & & & \\
\hline $5 / 2[752]-Q_{22}^{+}$ & 0.9 & 329.9 & 616.8 & 494.3 \\
\hline $1 / 2[501]$ & 78.8 & & & \\
\hline $1 / 2[770]$ & 5.1 & & & \\
\hline $5 / 2[503]-Q_{22}^{+}$ & 4.4 & & & \\
\hline $3 / 2[501]-Q_{22}^{+}$ & 3.7 & & & \\
\hline $1 / 2[640]+Q_{30}^{+}$ & 2.7 & & & \\
\hline $1 / 2[610]+Q_{30}^{+}$ & 1.0 & & $\sim 834$ & \\
\hline $1 / 2[631]$ & 55.1 & & & \\
\hline $1 / 2[651]$ & 7.9 & & & \\
\hline $1 / 2[640]$ & 6.7 & & & \\
\hline $1 / 2[500]+Q_{30}^{+}$ & 7.2 & & & \\
\hline $1 / 2[761]+Q_{30}^{+}$ & 5.8 & & & \\
\hline $1 / 2[770]+Q_{30}^{+}$ & 4.4 & & & \\
\hline $5 / 2[633]-Q_{22}^{+}$ & 2.5 & & $\sim 930$ & \\
\hline $5 / 2[503]$ & 87.2 & & & \\
\hline $5 / 2[752]$ & 1.7 & & & \\
\hline $1 / 2[500]+Q_{22}^{+}$ & 2.1 & & & \\
\hline $5 / 2[632]+Q_{30}^{+}$ & 0.9 & & $\sim 960$ & \\
\hline
\end{tabular}


2. The matrix elements of the operators $\hat{\jmath}_{+}, \hat{j}^{2}$, and $M(E, 1 \mu), M(E, 2 \mu), M(M, 1 \mu)$ were calculated, which were needed to construct the matrix of the total Hamiltonian and to calculate the transition probabilities.

3. The construction and diagonalization of the matrix of the total Hamiltonian $H$ was performed using the basis function (4). In the Coriolis mixing calculations we have included all functions $|I M K \rho\rangle$ with intrinsic states originating from the $i_{11 / 2}, g_{9 / 2}$ and $d_{5 / 2}$ shells (for positive parity) and from the $f_{9 / 2}, f_{5 / 2}$ and $j_{15 / 2}$ shells (for negative parity). The energies and wave functions of the levels of all rotational bands based on these intrinsic states were then obtained by diagonalization of the total Hamiltonian matrix for each value of the total angular momentum and parity. Since the QPM is microscopic without any free adjustable parameter for odd-A nuclei (all parameters are given by the eveneven core), the intrinsic energies obtained from this model are not expected to match exactly the experimental values. The QPM is 'microscopic' in the sense that it provides the two-quasiparticle structure of the core phonon, in contrast to phenomenological models in which the question of phonon structure is a priori excluded. Some discrepancies are also expected because the QPM is limited to 'one phonon' excitations and neglects multiphonon components in the wave function, which may be important in some cases (as in the transitional region) [38]. For this reason, the accuracy of the predictions of the QPM for the intrinsic energies of odd $-\mathrm{A}$ nuclei cannot be better than $\pm 50 \mathrm{keV}$ [31]. However, the Coriolis mixing amplitudes, $b_{K \rho}^{I q}$, in the rotational wave function (5), which are important for the calculation of transitional probabilities [Eq. (10)], are very sensitive to the position of the rotational bands in the spectrum. In order to make these Coriolis amplitudes more realistic, we tuned the QPM intrinsic energies with the requirement to obtain the rotational energies $E_{q}(I)$ [see Eq.(5)] as close to the experimental values as possible. The adopted (tuned) values of the intrinsic energies $E_{\rho}^{(\text {intr) }}$, which are presented in the fifth column of Tables 7 and 8 relative to the ground-state value $E_{\rho_{0}}^{(\mathrm{intr})}$, can be compared with the corresponding QPM results listed in column four. For the bands unobserved experimentally we used our calculated QPM values for the intrinsic energies without any tuning. Two more adjustable parameters were the inertia parameters $\hbar^{2}$ and 2ऽ: one common value of $\hbar^{2} / 2 \Im=7.04 \mathrm{keV}$ was taken for all positive-parity bands and one common value of $\hbar^{2} / 2 \Im=7.11$ for all negative-parity bands in ${ }^{227} \mathrm{Ra}$. For ${ }^{223} \mathrm{Ra}$, these parameters were 7.52 and $7.41 \mathrm{keV}$ for the positive- and negative-parity bands, respectively.

4. The wave functions (5) of rotational states and the intrinsic elements (11) determined by the QPM and Coriolis calculations were used to obtain $B(X L)$ transition rates. In the numerical calculation we took $Q_{0}=740 \mathrm{fm}^{2}$ for ${ }^{227} \mathrm{Ra}$ and $550 \mathrm{fm}^{2}$ for ${ }^{223} \mathrm{Ra}$, and $g_{s}=5.59$ (for protons), $g_{l}=1.0$ (for protons), $g_{s}=-3.82$ (for neutrons), $g_{l}=0.0$ (for neutrons), $g_{R}=Z / A=0.39$. The effective charges $e_{\mathrm{eff}}^{(i)}(E 1)$ and $e_{\mathrm{eff}}^{(i)}(E 2)$ were [31]: $e_{\mathrm{eff}}(E 1)=-0.40$ for neutrons and 0.61 for protons; $e_{\mathrm{eff}}(E 2)=0.003$ for neutrons and 0.99 for protons.

\section{$6 \quad$ Theoretical interpretation of ${ }^{227} \mathbf{R a}$}

Figure 7 shows a comparison of the experimental spectrum with the energies obtained from the Coriolis coupling calculations, $E_{q}$. Since there were nine adjustable parameters in the calculations (seven parameters for the intrinsic energies of the observed bands and two inertia parameters), the good agreement between the experimental and calculated energies must be viewed with caution. 


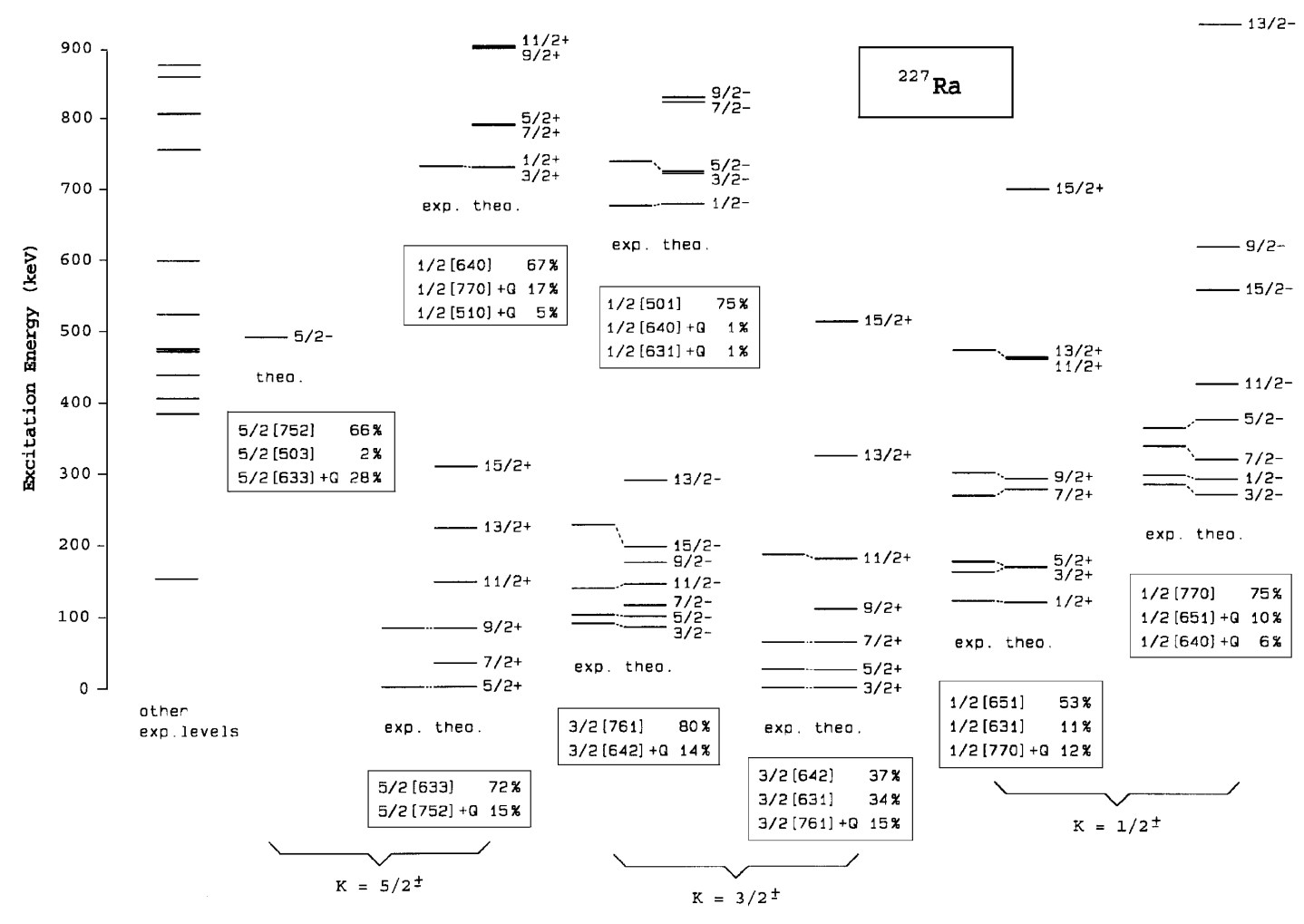

Figure 7: The energy spectra of the Coriolis coupling calculations and those found experimentally [19] for ${ }^{227} \mathrm{Ra}$.

Although the structure of the intrinsic state is given by two components - the dominant one-quasiparticle and the quasiparticle-plus-phonon components - it is the first of these that provides a direct matching between the QPM intrinsic states and the experimental bands. This is a particularly simple process since the bands have already been assigned the dominant Nilsson configurations on the basis of experimental properties, the systematics of the region, and comparison with the calculations [19]. Our calculations agree well with the previous assignments, except that the structure of the $K^{\pi}=3 / 2^{+}$ ground-state band, previously assigned as 3/2[631], appears more like a mixture of $3 / 2$ [642] and $3 / 2[631]$ in the present calculations. Similarly, the structure of the $K^{\pi}=1 / 2^{+}$band at $120.7 \mathrm{keV}$, previously assigned $1 / 2[631]$, is seen here as a mixture of a few components dominated by $1 / 2[651]$ and 1/2[631]. The importance of the quasiparticle-plus-phonon components is discussed next.

\subsection{Parity-doublet bands}

The theoretical spectrum provides not only the theoretical basis to classify but also the means to identify the PD bands of spin $K^{\pi}=1 / 2^{ \pm}, 3 / 2^{ \pm}, 5 / 2^{ \pm}$, and $7 / 2^{ \pm}$. The identification is provided by the strong octupole correlations present in the intrinsic structure of the two partner bands in each PD (see Table 7), supported further by proximity in the level energies. More specifically, one observes a symmetry in the intrinsic structure of the partner bands: the dominant one-quasiparticle component of the first partner coupled to the octupole phonon of the core forms the largest collective component in the structure of the second partner, and vice-versa. For example, the three main components of the $K^{\pi}=3 / 2^{+}$ground-state band are 3/2[642] (37\%), 3/2[631] (34\%), and 3/2[761] $+Q_{30}^{+}$ 
(15\%), while the intrinsic state of its PD partner is composed of mainly $3 / 2[761](80 \%)$ and $3 / 2[642]+Q_{30}^{+}(14 \%)$. In this way QPM reflects strong octupole correlations which are typical for this region of nuclei and which are responsible for the stable octupole deformation in ${ }^{222} \mathrm{Ra}$ and ${ }^{222,224} \mathrm{Th}[2,12]$.

Since matching of the calculated and experimental bands was based on the dominant one-quasiparticle components, the comparison of the predicted and observed PD partners (based on the collective components) becomes an independent process of model verification. The present calculations predict the 3/2[761] band to be the PD partner of the $3 / 2[642]+3 / 2[631]$ ground-state band, and the 1/2[770] band to be the PD partner of the $1 / 2[651]+1 / 2[631]$ band. These predictions are in full agreement with the adopted classification of the bands [19] and further confirmed by the new experimental data presented in Section 4.

Some of the predicted states have not been observed experimentally. There is no experimental evidence for the negative-parity partner to the $K^{\pi}=5 / 2^{+}$PD band identified at $1.7 \mathrm{keV}$. A possible candidate for the $K^{\pi}=5 / 2^{-}$bandhead, predicted in our calculations at about $\sim 500 \mathrm{keV}$, is the uninterpreted level at $598.5 \mathrm{keV}$. There is also no experimental evidence for the $K^{\pi}=7 / 2^{ \pm}$PD band theoretically predicted at $\sim 500 \mathrm{keV}$, but this could be attributed to the difficulty in accessing this band experimentally. A $K^{\pi}=7 / 2^{ \pm}$PD band at the energy of $\sim 100 \mathrm{keV}$ was also predicted in the reflection-asymmetric rotor calculations [9].

There are seven levels observed experimentally below $600 \mathrm{keV}$ [19] which are not involved in the theoretical doublet scheme presented in Fig. 7. Among these uninterpreted levels there are a few candidates for the bandheads of the second $K^{\pi}=3 / 2^{+}$and $K^{\pi}=$ $3 / 2^{-}$bands predicted in the calculations. However, the present calculations do not yield intrinsic states below $1 \mathrm{MeV}$ other than those listed in Table 7. In order to account for the other levels one needs to extend the QPM model to include other residual interaction (for example of the spin-multipole spin-multipole type). The model-calculated decoupling parameters are listed in Table 3, while the calculated magnetic moments for the groundstate $K^{\pi}=3 / 2^{ \pm}$band are $\mu_{3 / 2^{+}}=-0.15 \mathrm{~nm}$, and $\mu_{3 / 2^{-}}=-0.13 \mathrm{~nm}$. The measured value for the magnetic moment is $\mu_{3 / 2^{+}}=-0.4038(24) \mathrm{nm}$ [39].

\subsection{Enhancement of the B(E1) rates}

Table 4 provides a comparison of the experimental and calculated transition probabilities, $\gamma$-branching ratios and level half-lives. The first four columns list the energies of the initial and final levels, band assignment of the levels, and the connecting $\gamma$-transition for the experimentally observed levels which were interpreted in the present theoretical calculations (Fig. 7). The reduced transition probabilites, $B(X L ; i \rightarrow f)$, are given in columns six and seven for the multipolarity listed in column five. For the transitions where the $E 1+M 2$ mixing is allowed by the spin/parity selection rules, the experimental $B(E 1)$ rates are given by assuming pure $E 1$ transitions since no significant $E 1 / M 2$ mixing can be realistically expected. For the mixed $M 1+E 2$ transitions where the $E 2$ component is expected (or measured) to be significant, the $B(M 1)$ and $B(E 2)$ rates are given separately. In the case of the $182.4-$ and $204.3-\mathrm{keV}$ transitions, the $B(M 1)$ and $B(E 2)$ rates include the experimentally measured $\delta(M 1 / E 2)$ mixing ratio [19]. In other cases, the $B(M 1)$ (and similarly $B(E 2)$ ) rate is given by assuming that the contribution of the $M 1$ component is $\leq 100 \%$ (or equivalently, the $E 2$ component is $\leq 100 \%$ ). Columns eight and nine present the experimental and theoretical $\gamma$-branching ratios for transitions from a given initial state. These ratios are normalized to 100 for the strongest transition 
de-exciting a level. Finally, the last two columns yield the experimental and theoretical half-lives of a given initial level. The theoretical calculations yield only an upper limit of the half-life for those levels which are observed experimentally to de-excite to the uninterpreted levels. The detailed comparison of the theoretical and experimental results listed in Table 4 shows a good agreement between these results. Some aspects of this comparison are discussed below in more detail. The most important outcome of the calculations is the correct prediction of fast $B(E 1)$ rates.

The high octupole components in the intrinsic states lead in some cases to an enhancement of the $B(E 1)$ rates, particularly if the transition connects partner bands in a PD. This can be demonstrated more explicitly in the example of the 90-keV transition connecting the bandheads of the $K^{\pi}=3 / 2^{-}$and $3 / 2^{+}$PD bands with the main transition component of $\frac{3}{2} \frac{3}{2}[761] \rightarrow \frac{3}{2} \frac{3}{2}[642]$. Without any octupole collectivity, the standard Nilsson single-particle matrix element $\left\langle\frac{3}{2}[642]|M(E, 10)| \frac{3}{2}[761]\right\rangle$ is $\sim-0.0063 \mathrm{e} \cdot \mathrm{fm}$. With the octupole collectivity present, the intrinsic matrix element $\left\langle\Psi_{3 / 2}\left(\frac{3}{2}[642]\right)|M(E, 10)| \Psi_{3 / 2}\left(\frac{3}{2}[761]\right)\right\rangle$ calculated with the wave functions (7) (which have the structure given in Table 7 ) and Eq. (14) gives $\sim 0.023 \mathrm{e} \cdot \mathrm{fm}$. The main contribution to this matrix element is given by the third and fourth terms in Eq. (14). Thus, the enhancement of the $B(E 1)$ value for this transition relative to the Nilsson single-particle value is given by the factor of $(0.023 /-0.0063)^{2} \approx 13$. In this way the octupole phonon components provide a significant enhancement of the $B(E 1)$ values for the intra-PD band transitions, just as fast $E 1$ transitions are seen in nuclei with stable octupole deformations.

As seen in Table 4, the model calculations reproduce quite well the $E 1, M 1$, and $E 2$ transition rates for the $K^{\pi}=1 / 2^{ \pm}$and $3 / 2^{ \pm}$bands in ${ }^{227} \mathrm{Ra}$, including the individual characteristics of these PD bands. A good example of the close agreement between experiment and theory that was achieved for various types of $\gamma$-transitions is provided by the $B(E L)$ and $\gamma$-branchings for $\gamma$-rays de-exciting the $284.3-\mathrm{keV}$ level.

The comparison of the measured and calculated $E 1$ intra-PD band transitions is well illustrated in Table 6 using the $\left|D_{0}\right|$ parameters calculated from Eq. (1). The model predicts significantly higher $B(E 1)$ rates for the inter-PD transitions in the $K^{\pi}=1 / 2^{ \pm}$ band than in the $3 / 2^{ \pm}$band, which is consistent with the experimental data. For the $K^{\pi}=3 / 2^{ \pm} \mathrm{PD}$ band, it predicts a strong quenching of the $B\left(E 1 ; 5 / 2^{-} \rightarrow 5 / 2^{+}\right)$transition, and this effect is observed experimentally with precisely the same magnitude as calculated. On the other hand, no such effect was calculated for the $K^{\pi}=1 / 2^{ \pm}$band, in agreement with our experimental results.

In ${ }^{227} \mathrm{Ra}$, the intra- and inter-PD band transitions can no longer be classified as 'fast' and 'slow', respectively, since they are found to have strongly overlapping ranges. This is in agreement with the model calculations, which predict a wide range of the $B(E 1)$ values from $3 \times 10^{-3}$ down to $9 \times 10^{-7} \mathrm{e}^{2} \cdot \mathrm{fm}^{2}$ for the inter-PD band transitions in ${ }^{227} \mathrm{Ra}$ (see Table 4). The fastest rates $\left(1-3 \times 10^{-3} \mathrm{e}^{2} \cdot \mathrm{fm}^{2}\right)$ are predicted for the $E 1$ transitions from the $K^{\pi}=1 / 2^{+}$to the $K^{\pi}=3 / 2^{-}$bands. Unfortunately, these transitions have not been observed experimentally. Transitions from the $K^{\pi}=3 / 2^{-}$to the $K^{\pi}=5 / 2^{+}$bands are predicted with the rate of about $1 \times 10^{-4} \mathrm{e}^{2} \cdot \mathrm{fm}^{2}$, which is in qualitative agreement with the observed rates. The $E 1$ transitions from the $K^{\pi}=1 / 2^{-}$to the $K^{\pi}=3 / 2^{+}$bands are predicted to be slow in the range from $10^{-5}$ to $10^{-7} \mathrm{e}^{2} \cdot \mathrm{fm}^{2}$, and this is also in agreement with the observed transition rates and the $\gamma$-branching ratios. 
The same model calculations but with slightly modified parameters were performed for ${ }^{223} \mathrm{Ra}$. The selection of ${ }^{223} \mathrm{Ra}$ was motivated by the large body of experimental data describing this nucleus and by its structure. This nucleus is positioned in the centre of the octupole deformed region, and thus its structure could be contrasted with that of the octupole transitional ${ }^{227}$ Ra. Figure 8 illustrates the experimental and calculated results for the low-lying bands in ${ }^{223} \mathrm{Ra}$, which include the well known $K^{\pi}=1 / 2^{ \pm}, 3 / 2^{ \pm}$, and $5 / 2^{ \pm}$PD bands. The band energies for ${ }^{223} \mathrm{Ra}$ seem to be in better agreement with experimental values than those for ${ }^{227} \mathrm{Ra}$ (Fig. 7). However, the focus of this study is on the $E 1$ transition rates illustrated in Tables 4 and 5 .

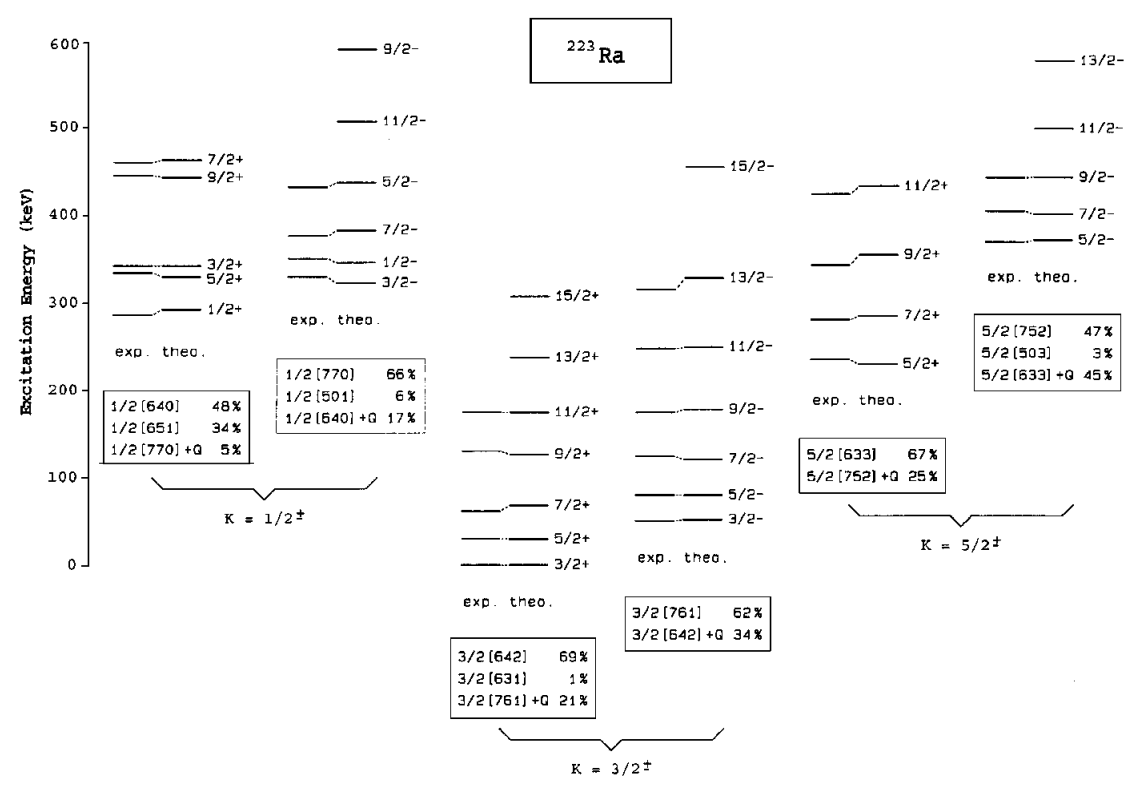

Figure 8: The energy spectra of the Coriolis-coupling calculations and those found experimentally [19] for ${ }^{223} \mathrm{Ra}$.

For the group of $19 E 1$ transition rates found experimentally, which consists of the intra- and inter-PD band transitions, about 15 are within a factor of $\sim 2$ of the calculated values. This represents a close agreement considering that the predicted $B(E 1)$ rates span slightly over four orders of magnitude, from $1.1 \times 10^{-7}$ to $2.0 \times 10^{-3} \mathrm{e}^{2} \cdot \mathrm{fm}^{2}$. There are four cases of strong disagreement, which are confined to the intra-PD band transitions for the $K^{\pi}=1 / 2^{ \pm}$and $3 / 2^{ \pm}$bands. In each case the model calculations significantly underpredict the observed $B(E 1)$ strength (by a factor of 3 to 7 ).

The results, best illustrated in Table 6 , indicate that the calculated intra-band transition rates for the $K^{\pi}=1 / 2^{ \pm}$band are consistently low which is in disagreement with the only known experimental value. More experimental results could clarify the issue and reveal whether the disagreement is confined to a single transition or to the whole PD band. The same table illustrates that, on average, the measured and calculated rates for the intra-PD band transitions for the $K^{\pi}=3 / 2^{ \pm}$band are almost equal. However, they followed opposite patterns: the calculated results show an increase in the transition rates when going from lower to higher spins of the parent state, while for the experimental results these rates decrease over the same range. Somewhat lower rates predicted for the 
$5 / 2^{-} \rightarrow 3 / 2^{+}, 5 / 2^{-} \rightarrow 5 / 2^{+}$, and $7 / 2^{-} \rightarrow 7 / 2^{+}$transitions are not in agreement with the experimental data. The calculations predict a strong quenching of the strength for the $9 / 2^{-} \rightarrow 9 / 2^{+}$transition - an interesting issue, as yet unresolved, and left for future measurements. Finally, the calculated results for the $K^{\pi}=5 / 2^{ \pm}$band are found to be in a close agreement with the experimental results. Considering the overall situation in ${ }^{223} \mathrm{Ra}$, the model calculations provide, on average, a good agreement with the experimental results. Note, for example, that the number of cases when the calculations overpredict and underpredict the experiment are almost equal. (Model calculations on some of the known levels in ${ }^{223} \mathrm{Ra}$ not discussed here are available from one of the authors: J. Kvasil.)

\section{$8 \quad$ Summary and conclusions}

The present study represents the first full application of the fast timing $\beta \gamma \gamma(\mathrm{t})$ method at PSB ISOLDE, combined with time calibrations at OSIRIS. Several level lifetimes were measured for the first time in ${ }^{227} \mathrm{Ra}$ revealing strong $E 1$ transitions connecting $\mathrm{PD}$ bands. The average strength of the intra-PD band transitions in the octupole transitional ${ }^{227} \mathrm{Ra}$ was found to be quite similar to that of the octupole deformed ${ }^{223} \mathrm{Ra}$. Unlike ${ }^{223} \mathrm{Ra}$, where the $B(E 1)$ rates for the intra- and inter-PD band transitions occupy two separate ranges, in ${ }^{227} \mathrm{Ra}$ they strongly overlap. This is due to a strong quenching of the $B(E 1)$ rates observed for some intra-PD transitions, and an enhancement of strength for a few inter-PD transitions. The experimental data provide evidence for the configuration dependence of the $\left|D_{0}\right|$ values in ${ }^{223} \mathrm{Ra}$ and ${ }^{227} \mathrm{Ra}$. Furthermore, the experimental $D_{0}$ values are found to be 2-3 times higher than predicted by the shell-correction method [2], both in the octupole deformed region and in the octupole transitional region.

In the theoretical part of this study we investigated the ability of the quasiparticleplus-phonon model with the inclusion of Coriolis coupling to interpret the results, and particularly the transition rates, for the octupole transitional ${ }^{227} \mathrm{Ra}$. This study was extended to also include, for the purpose of comparison, the octupole deformed ${ }^{223} \mathrm{Ra}$. It was the first time that this model had been used for the interpretation of the transition rates in the actinide region. The model, which is based on the weak-coupling scheme, provides a theoretical description of the octupole correlations via residual interactions and was used here to reveal highly collective octupole vibrational components of the low-lying states in these nuclei. We demonstrated, in the example of ${ }^{223} \mathrm{Ra}$ and ${ }^{227} \mathrm{Ra}$, that these octupole vibrational components cause intense dipole transitions between low-lying states, and their structure allows identification of the doublet character of the low-lying bands in a similar way to the strong-coupling scheme with a stable octupole deformation. The model calculations reproduce remarkably well the general enhancement (and occasional quenching) of the $E 1$ intra-doublet and some enhanced extra-doublet transitions in ${ }^{227}$ Ra. The results of similar calculations on ${ }^{223} \mathrm{Ra}$ were found to be in good overall agreement with the experimental data, although some systematic differences were observed. Some specific model predictions for ${ }^{223} \mathrm{Ra}$ and ${ }^{227} \mathrm{Ra}$, related to the strongly enhanced inter-doublet and strongly quenched intra-doublet $E 1$ transitions, remain to be verified experimentally.

\section{$9 \quad$ Acknowledgements}

This work was supported in part by the Norwegian Scientific Research Committee, the Nansen Foundation, the Swedish Natural Science Research Council, the CICYT 'Plan Nacional de Altas Energias' Spain (under contract AEN94-0833-C02-02), the UK Engineering and Physical Sciences Research Council, and the Polish Scientific Research 
Committee. A.J. Aas would also like to thank the OSIRIS group for their generous hospitality.

\section{References}

[1] J.L. Egido and L.M. Robledo, Nucl. Phys. A494, 85 (1989), and references therein.

[2] P.A. Butler and W. Nazarewicz, Nucl. Phys. A533, 249 (1991), and references therein.

[3] H. Mach, R.L. Gill, and M. Moszyński, Nucl. Instrum. Methods Phys. Res. A280, 49 (1989).

[4] M. Moszyński and H. Mach, Nucl. Instrum. Methods Phys. Res. A277, 407 (1989).

[5] H. Mach, F.K. Wohn, G. Molnár, K. Sistemich, J.C. Hill, M. Moszyński, R.L. Gill, W. Krips, and D.S. Brenner, Nucl. Phys. A523, 197 (1991).

[6] H. Mach, J. Billowes, M.J.G. Borge, D.G. Burke, P.A. Butler, J.F.C. Cocks, B. Fogelberg, S.J. Freeman, I.S. Grant, K. Gulda, G.D. Jones, E. Hagebø, P. Hoff, J. Hønsi, W. Kurcewicz, G. Løvhøiden, R.A. Naumann, K. Nybø, G. Nyman, H. Ravn, B. Rubio, J. Simpson, A.G. Smith, J.F. Smith, K. Steffensen, J.L. Tain, O. Tengblad, T.F. Thorsteinsen, and the ISOLDE Collaboration, in Nuclear Shapes and Nuclear Structure at Low Excitation Energy, eds. M. Vergnes, D. Goutte, P.H. Heenen, and J. Sauvage (Editions Frontières, Gif-sur-Yvette, 1994), p. 391.

[7] S. Ćwiok and W. Nazarewicz, Nucl. Phys. A529, 95 (1991).

[8] G.A. Leander and R.K. Sheline, Nucl. Phys. A413, 375 (1984).

[9] G.A. Leander and Y.S. Chen, Phys. Rev. C37, 2744 (1988).

[10] G.A. Leander and Y.S. Chen, Phys. Rev. C35, 1145 (1987).

[11] P. Bonche, P.H. Heenen, H. Flockard, and D. Vautherin, Phys. Lett. B175, 387 (1986).

[12] J.L. Egido and L.M. Robledo, Nucl. Phys. A518, 475 (1990).

[13] R.R. Chasman, Phys. Lett. B175, 254 (1986).

[14] I. Hřivnáčová, J. Kvasil, D. Nosek, and R.R. Sheline, Physica Scripta T56, 256 (1995).

[15] I. Hamamoto, J. Höller, and X.Z. Zhang, Phys. Lett. B229, 17 (1989).

[16] R. Piepenbring, Phys. Rev. C27, 2968 (1983).

[17] R.K. Sheline, Y.S. Chen, and G.A. Leander, Nucl. Phys. A486, 306 (1988).

[18] W. Kurcewicz et al., to be submitted to Nucl. Phys. A.

[19] E. Browne, Nuclear Data Sheets 65, 669 (1992).

[20] H. Mach, to be published.

[21] H. Mach and B. Fogelberg, Physica Scripta T56, 270 (1995).

[22] M.J.G. Borge, D.G. Burke, H. Gietz, P. Hill, N. Kaffrell, W. Kurcewicz, G. Løvhøiden, S. Matteson, R.A. Naumann, K. Nybø, G. Nyman, T. Bjørnstad, and the ISOLDE Collaboration, Nucl. Phys. A464, 189 (1987).

[23] T. Von Egidy, G. Barreau, H.G. Börner, W.F. Davidson, J. Larysz, D.D. Warner, P.H.M. van Assche, K. Nybø, T.F. Thorsteinsen, G. Løvhøiden, E.R. Flynn, J.A. Cizewski, R.K. Sheline, D. Decman, D.G. Burke, G. Sletten, N. Kaffrell, W. Kurcewicz, T. Bjørnstad, and G. Nyman, Nucl. Phys. A365, 26 (1981).

[24] H. Mach, D. Jerrestam, B. Fogelberg, M. Hellström, J.P. Omtvedt, K.I. Erokhina, and V.I. Isakov, Phys. Rev. C51, 500 (1995).

[25] K. Jain and A.K. Jain, At. Data Nucl. Data Tables 50, 269 (1992). 
[26] R.K. Sheline, D. Decman, K. Nybø, T.F. Thorsteinsen, G. Løvhøiden, E.R. Flynn, J.A. Cizewski, D.K. Burke, G. Sletten, P. Hill, N. Kaffrell, W. Kurcewicz, G. Nyman, and G. Leander, Phys. Lett. B133, 13 (1983).

[27] Ch. Briancon, S. Ćwiok, S.A. Eid, V. Green, W.D. Hamilton, C.F. Liang, and R.J. Walen, J. Phys. (UK) G16, 1735 (1990).

[28] F. Rösel, H.M. Fries, K. Alder, and H.C. Pauli, At. Data Nucl. Data Tables 21, 291 (1978).

[29] A. Abdul-Hadi, V. Barci, B. Weiss, H. Maria, and G. Ardisson, Phys. Rev. C47, 94 (1993).

[30] C.F. Liang, P. Paris, J. Kvasil, and R.K. Sheline, Phys. Rev. C44, 676 (1991).

[31] V.G. Soloviev, Theory of Complex Nuclei (Pergamon, Oxford, 1976).

[32] A. Bohr and B.R. Mottelson, Nuclear Structure, Vol. 2 (Benjamin, New York, 1975).

[33] A.I. Levon, J. de Boer, G. Graw, J. Kvasil, M. Loewe, V.D. Valnion, M. Würkner, H. Baltzer, C. Günther, J. Manns, U. Müller, and T. Weber, Nucl. Phys. A598, 11 (1996).

[34] V.G. Soloviev, Microscopic Theory of Nuclei (Moscow, NAUKA, 1989, in Russian).

[35] A.K. Jain, R.K. Sheline, P.C. Sood, and J. Jain, Rev. Mod. Phys. 62, 393 (1990).

[36] C.M. Lederer and V.S. Shirley, Table of the Isotopes, 7th ed. (Wiley, New York, 1978).

[37] P.C. Sood, D.M. Headly, and R.K. Sheline, At. Data Nucl. Data Tables 51, 273 (1992).

[38] R. Piepenbring, Z. Phys. A323, 341 (1986); see also V. Leandri and R. Piepenbring, Phys. Rev. C37, 2779 (1988).

[39] S.A. Ahmad, W. Klempt, R. Nuegart, E.W. Otten, P.-G. Reinhard, G. Ulm, and K. Wendt, Nucl. Phys. A483, 244 (1988). 